TRANSACTIONS OF THE

AMERICAN MATHEMATICAL SOCIETY

Volume 359, Number 3, March 2007, Pages 1161-1189

S 0002-9947(06)03994-8

Article electronically published on September 12, 2006

\title{
CLOSED FORM SUMMATION OF $C$-FINITE SEQUENCES
}

\author{
CURTIS GREENE AND HERBERT S. WILF
}

To David Robbins

Abstract. We consider sums of the form

$$
\sum_{j=0}^{n-1} F_{1}\left(a_{1} n+b_{1} j+c_{1}\right) F_{2}\left(a_{2} n+b_{2} j+c_{2}\right) \ldots F_{k}\left(a_{k} n+b_{k} j+c_{k}\right),
$$

in which each $\left\{F_{i}(n)\right\}$ is a sequence that satisfies a linear recurrence of degree $D(i)<\infty$, with constant coefficients. We assume further that the $a_{i}$ 's and the $a_{i}+b_{i}$ 's are all nonnegative integers. We prove that such a sum always has a closed form, in the sense that it evaluates to a linear combination of a finite set of monomials in the values of the sequences $\left\{F_{i}(n)\right\}$ with coefficients that are polynomials in $n$. We explicitly describe two different sets of monomials that will form such a linear combination, and give an algorithm for finding these closed forms, thereby completely automating the solution of this class of summation problems. We exhibit tools for determining when these explicit evaluations are unique of their type, and prove that in a number of interesting cases they are indeed unique. We also discuss some special features of the case of "indefinite summation", in which $a_{1}=a_{2}=\cdots=a_{k}=0$.

\section{INTRODUCTION}

In Section 1.6 of [7] the following assertion is made:

All Fibonacci number identities such as Cassini's $F_{n+1} F_{n-1}-F_{n}^{2}=$ $(-1)^{n}$ (and much more complicated ones), are routinely provable using Binet's formula:

$$
F_{n}:=\frac{1}{\sqrt{5}}\left(\left(\frac{1+\sqrt{5}}{2}\right)^{n}-\left(\frac{1-\sqrt{5}}{2}\right)^{n}\right) .
$$

This is followed by a brief Maple program that proves Cassini's identity by substituting Binet's formula on the left side and showing that it then reduces to $(-1)^{n}$. Another method of proving these identities is given in 10, in which it is observed that one can find the recurrence relations that are satisfied by each of the two sides of the identity in question, show that they are the same and that the initial values agree, and the identity will then be proved.

The purpose of this note is to elaborate on these ideas by showing how to derive, instead of only to verify, summation identities for a certain class of sequence sums, and to show that this class of sums always has closed form in a certain sense, and

Received by the editors May 20, 2004, and, in revised form, December 9, 2004.

2000 Mathematics Subject Classification. Primary 05A15, 05A19; Secondary 11B37, 11 B39.

$K e y$ words and phrases. Summation, closed form, $C$-finite, recurrences. 
that these closed forms can be found entirely algorithmically. Indeed, a Mathematica program that carries out the procedures that we develop in this paper can be downloaded from the web sites of the authors [4.

We deal with the class of $C$-finite sequences (see [10]). These are the sequences $\{F(n)\}_{n \geq 0}$ that satisfy linear recurrences of fixed span with constant coefficients. The Fibonacci numbers, e.g., will do nicely for a prototype sequence of this kind. The kind of sum that we will consider first will be of the form (2) below. We will say that such a sum has an $F$-closed form if there is a linear combination of a fixed (i.e., independent of $n$ ) number of monomials in values of the $F$ 's such that for all $n$ the sum $f(n)$ is equal to that linear combination.

For example, consider the sum

$$
f(n)=\sum_{j=0}^{n-1} F(j)^{2} F(2 n-j)
$$

where the F's are Fibonacci numbers. In Section 3.1 we will see how to use our method to show that $f(n)$ can be expressed in the form (11), which is a linear combination of five monomials in the F's. Hence the sum $f(n)$ has an $F$-closed form.

More generally, we will consider functions $F(n)$ satisfying a recurrence of minimal order $D$ with constant coefficients, whose associated polynomial has roots $r_{1}, r_{2}, \ldots, r_{d}$, of multiplicities $e_{1}, e_{2}, \ldots, e_{d}$, where $\sum_{i} e_{i}=D$. Such a function may be expressed in the form

$$
F(n)=\sum_{m=1}^{d} \sum_{h=0}^{e_{m}-1} \lambda_{m, h} n^{h}\left(r_{m}\right)^{n},
$$

where the $r_{i}$ are distinct and nonzero, and $\lambda_{m, e_{m}-1} \neq 0$ for all $m$.

We will begin by evaluating sums of the form

$$
f(n)=\sum_{j=0}^{n-1} F\left(a_{1} n+b_{1} j+c_{1}\right) \cdots F\left(a_{k} n+b_{k} j+c_{k}\right)
$$

in which the $a$ 's, $b$ 's, and $c$ 's are given integers. We assume further that, for all $i$, $a_{i} \geq 0$ and $a_{i}+b_{i} \geq 0$ and at least one of these is positive. Later we will generalize this result to allow the $F$ 's in the summand to be different $C$-finite functions. The principal result of this paper is perhaps Theorem 17 below, which proves in full generality, i.e., with arbitrary root multiplicities and with the $F$ 's in the summand all being different, the existence of closed forms, and exhibits an explicit finite basis for the solution space.

It is elementary and well known that $f(n)$ is $C$-finite, and one can readily obtain explicit expressions for $f(n)$ in terms of the roots $r_{m}$. Our first results show how to obtain formulæ for sums $f(n)$ of the form (2) as a polynomial in the $F$ 's, based on two different explicit sets of "target" monomials in the $F$ 's. Using the first target set, we obtain the following result.

Theorem 1. The sum $f(n)$ in (2) has an F-closed form. It is equal to a linear combination of monomials in the F's, of the form

$$
\begin{array}{cl}
F\left(\left(a_{1}+b_{1}\right) n+i_{1}\right) \ldots F\left(\left(a_{k}+b_{k}\right) n+i_{k}\right), & 0 \leq i_{\nu} \leq D-1, \\
\psi_{i_{1}, \ldots, i_{k}}(n) F\left(a_{1} n+i_{1}\right) F\left(a_{2} n+i_{2}\right) \ldots F\left(a_{k} n+i_{k}\right), & 0 \leq i_{\nu} \leq D-1,
\end{array}
$$


where $\psi_{i_{1}, \ldots, i_{k}}(n)$ denotes a polynomial of degree at most $\beta=1+\Delta \mu$, where $\Delta=$ $\max _{m}\left\{e_{m}-1\right\}$ and $\mu=\left|\left\{i: a_{i}=0\right\}\right|$. If $F$ is rational-valued, then there are solutions in which all coefficients to be determined are rational.

We note that, if the roots $r_{i}$ are distinct, then $\beta=1$ and the polynomials $\psi_{i_{1}, \ldots, i_{k}}(n)$ are linear. The next theorem gives a closed form in terms of an alternate target set of monomials.

Theorem 2. The sum $f(n)$ in (2) can be expressed in F-closed form as a linear combination of monomials of the form

$$
\begin{array}{clrl}
F\left(n+i_{1}\right) F\left(n+i_{2}\right) \ldots F\left(n+i_{P}\right), & 0 \leq i_{\nu} \leq D-1, \\
\psi_{i_{1}, \ldots, i_{k}}(n) F\left(n+i_{1}\right) F\left(n+i_{2}\right) \ldots F\left(n+i_{Q}\right), & 0 \leq i_{\nu} \leq D-1,
\end{array}
$$

where $P=\left(a_{1}+b_{1}\right)+\left(a_{2}+b_{2}\right)+\cdots+\left(a_{k}+b_{k}\right), Q=a_{1}+a_{2}+\cdots+a_{k}$, and $\psi_{i_{1}, \ldots, i_{k}}(n)$ denotes a polynomial of degree $\gamma=\max \left\{0,1+\Delta\left(k-\sum\left\{a_{i} \mid a_{i}>0\right\}\right)\right\}$. If $F$ is rational-valued, then there are solutions in which all coefficients to be determined are rational.

For example, when $F(n)$ is the $n$th Fibonacci number, Theorem 2 states that any sum of the form (2) can be expressed as a linear combination of monomials in $F(n)$ and $F(n+1)$, with rational linear polynomial coefficients, where those monomials have at most two distinct degrees. Again, we note that, if the roots $r_{i}$ are distinct, then $\gamma=1$ and the polynomials $\psi_{i_{1}, \ldots, i_{k}}(n)$ are linear.

The natural domain for these questions is the vector space $\mathbf{V}^{\infty}$ of complexvalued functions on $\{0,1,2, \ldots\}$. However, to obtain our expansions it is only necessary to work in the vector space $\mathbf{V}^{M}$ of functions on $\{1, \ldots, M\}$, where $M$ is the number of unknown coefficients to be determined. More precisely, we define $M$ to be equal to the number of "algebraically distinct" monomials of the form $n^{h} \prod_{\nu} F\left(\left(a_{\nu}+b_{\nu}\right) n+i_{\nu}\right)$ or $n^{h} \prod_{\nu} F\left(a_{\nu} n+i_{\nu}\right)$ generated by (3) or (4). Here we consider two monomials to be equivalent if they differ by a rearrangement of factors, or by a constant factor arising from cases where $a_{\nu}+b_{\nu}=0$ or $a_{\nu}=0$. (For examples, see Section 3.) Then we have the following result.

Theorem 3. Let $\mathbf{W}^{M} \subseteq \mathbf{V}^{M}$ be the vector space of complex-valued functions on $\{1,2, \ldots, M\}$ spanned by the monomials in (3), where $M$ is the number of algebraically distinct monomials generated by (3), as defined in the previous paragraph. Let $\mathbf{W}^{\infty} \subseteq \mathbf{V}^{\infty}$ be the vector space of functions on $\{0,1, \ldots\}$ spanned by the same monomials. If two linear combinations of monomials of type (3) agree in $\mathbf{W}^{M}$, then they agree in $\mathbf{W}^{\infty}$. A similar statement holds for monomials of type (4).

As a consequence, we can obtain expressions of type (3) or (4) by equating $M$ values of $f(n)$ to the values of the assumed linear combinations, and solving for the coefficients. We note that $M \leq(\beta+2) d^{k}$ in case (3) and $M \leq d^{P}+(\gamma+1) d^{Q}$ in case (44).

In general, $F$-closed expressions are not unique. For example, we may add terms of the form $\Psi(F)(F(n+2)-F(n+1)-F(n))$, where $\Psi(F)$ is any polynomial in the $F(a n+i)$, to an expression involving Fibonacci numbers and get another valid $F$-closed form. However, the formats described by (3) and (4) are highly restrictive, and the resulting expressions can be shown to be unique in a surprising number of cases. We will return to the question of uniqueness and, more generally, to the problem of computing $\operatorname{dim}\left(\mathbf{W}^{\infty}\right)$, in Sections 4-6. 
The structure of this paper is as follows. In Sections 2-6 we consider the case where the roots $r_{i}$ all have multiplicity one. In this case, both the statements and proofs of our results are considerably simpler, and will serve as models for the more general case to be presented later. Section 2 gives proofs of Theorems 1, 2 and 3 in this special case. Section 3 illustrates these results with several examples. Sections 4-6 consider the issue of uniqueness and dimension, again for the distinct root case. Section 7 drops the assumption of distinct roots, and gives a proof of Theorems 11 and 2 in a more general form (Theorem 17) where the factors in the summand of (2) may involve different $F$ 's. Section 8 considers some issues that arise when $a_{1}=a_{2}=\cdots=a_{k}=0$, i.e., when the problem of computing $f(n)$ is an indefinite summation problem. Section 9 contains more examples.

\section{Proofs in the CASe of Distinct Roots}

In this section, we will assume that $F(n)$ satisfies a recurrence of minimal order $d$, with distinct roots, and hence can be expressed in the form

$$
F(n)=\sum_{m=1}^{d} \lambda_{m} r_{m}^{n}
$$

with the $r_{m}$ distinct and the $\lambda_{m}$ nonzero. Expanding the right side of (2) above and using (5), we find that

$$
f(n)=\sum_{j=0}^{n-1} \prod_{\ell=1}^{k}\left\{\sum_{m=1}^{d} \lambda_{m} r_{m}^{a_{\ell} n+b_{\ell} j+c_{\ell}}\right\} .
$$

A typical term in the expansion of the product will look like

$$
K r_{m_{1}}^{a_{1} n+b_{1} j+c_{1}} r_{m_{2}}^{a_{2} n+b_{2} j+c_{2}} \ldots r_{m_{k}}^{a_{k} n+b_{k} j+c_{k}},
$$

in which $K$ is a constant, i.e., is independent of $n$ and $j$, which may be different at different places in the exposition below. Since we are about to sum the above over $j=0, \ldots, n-1$, put

$$
\Theta=r_{m_{1}}^{b_{1}} r_{m_{2}}^{b_{2}}, \ldots, r_{m_{k}}^{b_{k}}
$$

because this is the quantity that is raised to the $j$ th power in the expression (6). Now there are two cases, namely $\Theta=1$ and $\Theta \neq 1$.

Suppose $\Theta=1$. Then the sum of our typical term (6) over $j=0, \ldots, n-1$ is

$$
K n\left(r_{m_{1}}^{a_{1}} r_{m_{2}}^{a_{2}} \ldots r_{m_{k}}^{a_{k}}\right)^{n}
$$

On the other hand, if $\Theta \neq 1$, then the sum of our typical term (6) over $j=$ $0, \ldots, n-1$ is

$$
K\left\{\left(r_{m_{1}}^{a_{1}+b_{1}} \ldots r_{m_{k}}^{a_{k}+b_{k}}\right)^{n}-\left(r_{m_{1}}^{a_{1}} \ldots r_{m_{k}}^{a_{k}}\right)^{n}\right\} .
$$

The next task will be to express these results in terms of various members of the sequence $\{F(n)\}$ instead of in terms of various powers of the $r_{i}$ 's. To do that we write out (5) for $d$ consecutive values of $n$, getting

$$
\begin{aligned}
F(n+i) & =\sum_{m=1}^{d} \lambda_{m} r_{m}^{n+i} \quad(i=0,1, \ldots, d-1) \\
& =\sum_{m=1}^{d}\left(\lambda_{m} r_{m}^{i}\right) r_{m}^{n} \quad(i=0,1, \ldots, d-1) .
\end{aligned}
$$


We regard these as $d$ simultaneous linear equations in the unknowns $\left\{r_{1}^{n}, \ldots, r_{d}^{n}\right\}$, with a coefficient matrix that is a nonsingular diagonal matrix times a Vandermonde based on distinct points, and is therefore nonsingular. Hence for each $m=1, \ldots, d$, $r_{m}^{n}$ is a linear combination of $F(n), F(n+1), \ldots, F(n+d-1)$, with coefficients that are independent of $n$. Thus in (7), (8) we can replace each $r_{m_{i}}^{n a_{i}}$ by a linear combination of $F\left(a_{i} n\right), F\left(a_{i} n+1\right), \ldots, F\left(a_{i} n+d-1\right)$, and we can replace each $r_{m_{i}}^{n\left(a_{i}+b_{i}\right)}$ by a linear combination of

$$
F\left(\left(a_{i}+b_{i}\right) n\right), F\left(\left(a_{i}+b_{i}\right) n+1\right), \ldots, F\left(\left(a_{i}+b_{i}\right) n+d-1\right) .
$$

After making these replacements, we see that the two possible expressions (7), (8) contribute monomials that are all of the form (3), with the polynomials $\psi_{i_{1}, \ldots, i_{k}}$ all linear. This establishes the existence of expansions in monomials of type (3) , as claimed in Theorem 1.

To prove the corresponding claim made in Theorem 2, it suffices to observe that, in the above argument, we could have written $r_{m_{i}}^{n a_{i}}=\left(r_{m_{i}}^{n}\right)^{a_{i}}$ and replaced it by a homogeneous polynomial of degree $a_{i}$ in $F(n), F(n+1), \ldots, F(n+d-1)$. Similar reasoning applies to $r_{m_{i}}^{n\left(a_{i}+b_{i}\right)}$. Thus all of the resulting monomials are of type (4).

We continue now with the proof of Theorem 3 . The arguments are identical for cases (3) and (4), so we will consider only case (3). We have observed that for each $i=0, \ldots, d-1, F(n+i)$ is a linear combination of $r_{1}^{n}, \ldots, r_{d}^{n}$, and conversely. Hence, in both $\mathbf{V}^{M}$ and $\mathbf{V}^{\infty}$, the linear span of the set

$$
F\left(a_{1} n+i_{1}\right) F\left(a_{2} n+i_{2}\right) \ldots F\left(a_{k} n+i_{k}\right), \quad 0 \leq i_{\nu} \leq d-1,
$$

is equal to the linear span of the set $\left\{\theta_{1}^{n}, \theta_{2}^{n}, \ldots, \theta_{d^{k}}^{n}\right\}$, where the $\theta_{j}$ range over all monomials of the form

$$
r_{m_{1}}^{a_{1}} r_{m_{2}}^{a_{2}} \cdots r_{m_{k}}^{a_{k}}
$$

Arguing similarly for the other cases, we see that the linear span of all monomials of type (3) is equal to the linear span of the set of $3 d^{k}$ functions

$$
\begin{gathered}
\theta_{1}^{n}, \theta_{2}^{n}, \ldots, \theta_{d^{k}}^{n}, \\
\psi_{1}^{n}, \psi_{2}^{n}, \ldots, \psi_{d^{k}}^{n}, \\
n \psi_{1}^{n}, n \psi_{2}^{n}, \ldots, n \psi_{d^{k}}^{n},
\end{gathered}
$$

where the $\theta_{i}$ are as defined above and the $\psi_{j}$ range over all monomials of the form

$$
r_{m_{1}}^{a_{1}+b_{1}} r_{m_{2}}^{a_{2}+b_{2}} \cdots r_{m_{k}}^{a_{k}+b_{k}}
$$

We claim that the number of distinct functions appearing in (9) is less than or equal to $M$. Indeed, it is straightforward to check that the map $F(\theta n+i) \mapsto\left(r_{i+1}^{\theta}\right)^{n}$ extends to a well-defined, surjective map from the set of equivalence classes of monomials of type (3) to the set of functions appearing in (9).

Now suppose that $\Phi(n)$ and $\Psi(n)$ are linear combinations of monomials of type (3), with $\Phi(n)=\Psi(n)$ for $n=1,2, \ldots, M$. We know that $\Phi(n)$ and $\Psi(n)$ can both be expressed in the form

$$
\sum_{i} c_{i} \theta_{i}^{n}+\sum_{j} d_{j} \psi_{j}^{n}+\sum_{k} e_{k} n \psi_{k}^{n}
$$

for some constants $c_{i}, d_{j}, e_{k}$, where the sum is over distinct elements of (9) and hence there are at most $M$ terms in the sum. It follows from standard results in the 
theory of difference equations (e.g., see [3], Chapter 11) that $\Phi(n)$ and $\Psi(n)$ satisfy the same linear recurrence of order at most $M$ with constant coefficients, e.g., the recurrence with characteristic polynomial $\prod_{i}\left(t-\theta_{i}\right) \prod_{j}\left(t-\psi_{j}\right)^{2}$. Hence the values of $\Phi(n)$ and $\Psi(n)$ are completely determined by their values for $n=1,2, \ldots, M$, and since they agree for these values, they must agree for all $n$. This completes the proof of Theorem 3 ,

The proof also shows that the $C$-finite degree of $f(n)$ is bounded by $3 d^{k}$. Sharper bounds appear in Corollaries 19 and 20 below.

\section{EXAMPLES}

3.1. A Fibonacci sum. This work was started when a colleague asked about the sum

$$
f(n)=\sum_{j=0}^{n-1} F(j)^{2} F(2 n-j),
$$

in which the F's are the Fibonacci numbers. If we refer to the general form (2) of the question, we see that in this case

$$
k=3 ; d=2 ;\left(a_{1}, b_{1}, c_{1}\right)=\left(a_{2}, b_{2}, c_{2}\right)=(0,1,0) ;\left(a_{3}, b_{3}, c_{3}\right)=(2,-1,0) .
$$

If we now refer to the general form (3) of the answer we see that the sum $f(n)$ is a linear combination of monomials

$$
\begin{gathered}
n F(2 n), F(2 n), n F(2 n+1), F(2 n+1), F(n)^{3}, \\
F(n)^{2} F(n+1), F(n) F(n+1)^{2}, F(n+1)^{3} .
\end{gathered}
$$

Hence we assume a linear combination of these monomials and equate its values to those of $f(n)$ for $n=0,1, \ldots, 7$ to determine the constants of the linear combination. The result is that

$$
f(n)=\frac{1}{2}\left(F(2 n)+F(n)^{2} F(n+1)-F(n) F(n+1)^{2}+F(n+1)^{3}-F(2 n+1)\right) .
$$

This formula is expressed in terms of monomials of type (3). Using monomials of type (4), we obtain the alternate expression

$$
\begin{aligned}
f(n)=\frac{1}{2}\left(2 F(n) F(n+1)-2 F(n)^{2}-F(\right. & n+1)^{2}+F(n)^{2} F(n+1) \\
& \left.-F(n) F(n+1)^{2}+F(n+1)^{3}\right) .
\end{aligned}
$$

In Section 5 we will show that both of these expression are unique, i.e., (11) is the unique $F$-closed formula for $f(n)$ of type (3) and (12) is the unique $F$-closed formula of type (4).

3.2. An example involving subword avoidance. Given an alphabet of $A \geq 2$ letters, let $W$ be some fixed word of three letters such that no proper suffix of $W$ is also a proper prefix of $W$. For example, $W=a a b$ will do nicely. Let $G(n)$ be the number of $n$-letter words over $A$ that do not contain $W$ as a subword. It is well 
known, and obvious, that

$$
G(n)=A G(n-1)-G(n-3),
$$

with $G(0)=1, G(1)=A, G(2)=A^{2}$, so this is a $C$-finite sequence. It is easy to check that the roots of its associated polynomial equation are distinct for all $A \geq 2$. Suppose we want to evaluate the sum $g(n)=\sum_{j=0}^{n-1} G(j)^{2}$. Then

$$
k=2 ; d=3 ;\left(a_{1}, b_{1}, c_{1}\right)=\left(a_{2}, b_{2}, c_{2}\right)=(0,1,0) .
$$

Using either Theorem 1 or Theorem 2, we see that $g(n)$ is a linear combination of the monomials

$1, n, G(n)^{2}, G(n) G(n+1), G(n) G(n+2), G(n+1) G(n+2), G(n+1)^{2}, G(n+2)^{2}$.

As before, we assume a linear combination of these monomials with constants to be determined, and we equate the result to computed values of $g(n)$, for $n=0,1, \ldots, 7$, to solve for the constants. The end result is that

$$
\begin{aligned}
g(n)= & \frac{1}{A(A-2)}\left(1-(A-1)^{2} G(n)^{2}-2 G(n) G(n+1)+2 G(n) G(n+2)\right. \\
& \left.+2(A-1) G(n+1) G(n+2)-(A-1)^{2} G(n+1)^{2}-G(n+2)^{2}\right)
\end{aligned}
$$

if $A>2$, and

$$
\begin{aligned}
g(n)=n+2 G(n)^{2}+ & 7 G(n) G(n+1)-5 G(n) G(n+2) \\
& -5 G(n+1) G(n+2)+3 G(n+1)^{2}+2 G(n+2)^{2}
\end{aligned}
$$

if $A=2$.

In the case $A=2$, it is easy to show that $G(n)=F(n+3)-1$ for all $n$, where $F(n)$ is the $n$th Fibonacci number. Consequently, $G(n+2)-G(n+1)-G(n)=1$, and adding any multiple of the relation

$$
(G(n+2)-G(n+1)-G(n)-1)^{2}=0
$$

to the right side of (15) gives another degree 2 expression of type (3) or (4). Thus formula (15) is not unique within the class of formulæ of type (3) or (4). However, in Section 5 we will show that, when $A>2$, formula (14) is unique within this class. When $A=2$, we show that all relations are constant multiples of (16).

3.3. Fibonacci power sums. Theorems 1 and 2 imply that if the $F(j)$ 's are the Fibonacci numbers, then for each integer $p=1,2, \ldots$ there is a formula

$$
f(n)=\sum_{j=0}^{n-1} F(j)^{p}=\sum_{j=0}^{p} \Lambda_{p, j} F(n)^{j} F(n+1)^{p-j}+c_{p} n+d_{p} .
$$


Here is a brief table of values of these coefficients.

\begin{tabular}{r|rrrrrrrrrr}
$p$ & $\Lambda_{p, 0}$ & $\Lambda_{p, 1}$ & $\Lambda_{p, 2}$ & $\Lambda_{p, 3}$ & $\Lambda_{p, 4}$ & $\Lambda_{p, 5}$ & $\Lambda_{p, 6}$ & $\Lambda_{p, 7}$ & $c_{p}$ & $d_{p}$ \\
\hline 1 & 1 & 0 & 0 & 0 & 0 & 0 & 0 & 0 & 0 & -1 \\
2 & 0 & 1 & -1 & 0 & 0 & 0 & 0 & 0 & 0 & 0 \\
3 & $-\frac{1}{2}$ & $\frac{3}{2}$ & 0 & $-\frac{3}{2}$ & 0 & 0 & 0 & 0 & 0 & $\frac{1}{2}$ \\
4 & 0 & $\frac{2}{25}$ & $-\frac{3}{25}$ & $\frac{14}{25}$ & $-\frac{19}{25}$ & 0 & 0 & 0 & $\frac{6}{25}$ & 0 \\
5 & $\frac{7}{22}$ & $-\frac{5}{22}$ & $-\frac{15}{11}$ & $\frac{10}{11}$ & $\frac{15}{11}$ & $-\frac{15}{22}$ & 0 & 0 & 0 & $-\frac{7}{22}$ \\
6 & 0 & $\frac{1}{2}$ & $-\frac{5}{4}$ & 0 & $\frac{5}{4}$ & $\frac{1}{2}$ & -1 & 0 & 0 & 0 \\
7 & $-\frac{139}{638}$ & $\frac{763}{638}$ & $-\frac{945}{638}$ & $-\frac{350}{319}$ & $\frac{105}{58}$ & $\frac{357}{319}$ & $-\frac{105}{319}$ & $-\frac{777}{638}$ & 0 & $\frac{139}{638}$
\end{tabular}

The resulting expressions for $f(n)$ turn out to be unique within the class of type (31) or (4) formulæ when $p \not \equiv 0 \bmod 4$. When $p$ is a multiple of 4 (for example, in the fourth line of the table above) the formulæ are not unique, but are subject to a one-parameter family of relations generated by powers of the degree- 4 relation

$$
\left(F(n+1)^{2}-F(n)^{2}-F(n) F(n+1)\right)^{2}=1 .
$$

We will establish these facts in Section 5 .

3.4. Generic power sums. Consider the power sum

$$
f(n)=\sum_{k=0}^{n-1} F(k)^{2},
$$

where $F(n)$ solves a linear recurrence

$$
F(n)=A F(n-1)+B F(n-2)
$$

with initial values $F(0)$ and $F(1)$, where $A$ and $B$ are sufficiently general to insure that, if $r_{1}$ and $r_{2}$ are the associated roots, then $r_{1}$ and $r_{2}$ are distinct and none of the monomials $r_{1}^{2}, r_{1} r_{2}$, and $r_{2}^{2}$ equals 1 . This is equivalent to assuming simply that $A^{2}+4 B \neq 0, A \neq \pm(B-1)$, and $B \neq-1$.

Using techniques introduced earlier, we can express $f(n)$ as a linear combination of $F(n)^{2}, F(n) F(n+1), F(n+1)^{2}$, and 1 . The solution may be computed explicitly in terms of $A, B, F(0)$, and $F(1)$, and we find that $f(n)$ equals

$$
\frac{\left(1-B-A^{2}(1+B)\right) F(n)^{2}+(2 A B) F(n) F(n+1)+(1-B) F(n+1)^{2}-K}{\left(A^{2}-(B-1)^{2}\right)(B+1)},
$$

where

$$
K=\left(1-B-A^{2}(B+1)\right) F(0)^{2}+(2 A B) F(0) F(1)+(1-B) F(1)^{2} .
$$

In (17), we observe a curious phenomenon: since $F(n)$ depends on $F(0)$ and $F(1)$, we might expect that our linear equations would have led to a solution in which each of the coefficients depends on $F(0)$ and $F(1)$. However, this dependence appears only in the constant term. The next theorem demonstrates that such behavior is 
typical for power sums of $C$-finite functions in which the terms in (3) containing $n$ are not present, i.e., in cases where no monomial in the roots equals 1.

Theorem 4. Suppose that $\{F(n)\}_{n \geq 0}$ is a $C$-finite sequence determined by a recurrence of order $d$ together with initial conditions $F(0), F(1), \ldots, F(d-1)$. Suppose that the recurrence polynomial has distinct roots $r_{1}, \ldots, r_{d}$, and suppose that no monomial of degree $p$ in the $r_{i}$ equals 1 . Let $f(n)=\sum_{j=0}^{n-1} F(j)^{p}$, where $p$ is a positive integer, and let

$$
f(n)=\sum_{0 \leq i_{1}, i_{2}, \ldots, i_{d} \leq d-1} \Lambda_{i_{1}, i_{2}, \ldots, i_{d}} F\left(n+i_{1}\right) F\left(n+i_{2}\right) \cdots F\left(n+i_{d}\right)+K
$$

be the expansion of $f(n)$ obtained according to the method given in Section 2. Then the coefficients $\Lambda_{i_{1}, i_{2}, \ldots, i_{d}}$ in (18) do not depend on $F(0), F(1), \ldots, F(d-1)$.

Proof. Suppose that $F(n)=\sum_{m=1}^{d} \lambda_{m} r_{m}^{n}$. Define

$$
\mathbf{X}(n)=\left(\begin{array}{c}
\lambda_{1} r_{1}^{n} \\
\lambda_{2} r_{2}^{n} \\
\vdots \\
\lambda_{d} r_{d}^{n}
\end{array}\right) \quad \text { and } \quad \mathbf{Y}(n)=\left(\begin{array}{c}
F(n) \\
F(n+1) \\
\vdots \\
F(n+d-1)
\end{array}\right)
$$

Then we have

$$
\mathbf{Y}(n)=\mathbf{V} \mathbf{X}(n) \text { and } \mathbf{X}(n)=\mathbf{V}^{-1} \mathbf{Y}(n),
$$

where $\mathbf{V}$ is a Vandermonde matrix in the $r_{i}$. It follows from (19) that the terms $\lambda_{m} r_{m}^{n}, 1 \leq m \leq d$, can be expressed as linear combinations of the functions $F(n+i)$, with coefficients that do not depend on $F(0), F(1), \ldots, F(d-1)$. Using the method of Section 2, we can compute

$$
\begin{aligned}
f(n)=\sum_{j=0}^{n-1} F(j)^{p} & =\sum_{j=0}^{n-1}\left(\sum_{m=0}^{d} \lambda_{m} r_{m}^{j}\right)^{p} \\
& =\sum_{j=0}^{n-1}\left(\sum_{0 \leq i_{1}, i_{2}, \ldots, i_{p} \leq d} \lambda_{i_{1}} \lambda_{i_{2}} \cdots \lambda_{i_{p}}\left(r_{i_{1}} r_{i_{2}} \cdots r_{i_{p}}\right)^{j}\right) \\
& =\sum_{0 \leq i_{1}, i_{2}, \ldots, i_{p} \leq d} \lambda_{i_{1}} \lambda_{i_{2}} \cdots \lambda_{i_{p}} \frac{\left(r_{i_{1}} r_{i_{2}} \cdots r_{i_{p}}\right)^{n}-1}{\left(r_{i_{1}} r_{i_{2}} \cdots r_{i_{p}}\right)-1} \\
& =\sum_{0 \leq i_{1}, i_{2}, \ldots, i_{p} \leq d} \frac{\left(\lambda_{i_{1}} r_{i_{1}}^{n}\right)\left(\lambda_{i_{2}} r_{i_{2}}^{n}\right) \cdots\left(\lambda_{i_{p}} r_{i_{p}}^{n}\right)}{\left(r_{i_{1}} r_{i_{2}} \cdots r_{i_{p}}\right)-1}-K,
\end{aligned}
$$

where $K$ is a constant. Using (19), we can express all of the terms in (20) except $K$ as a linear combination of monomials in the $F(n+i)$ with coefficients that do not depend on $F(0), F(1), \ldots, F(d-1)$, as claimed.

We will return to this subject in Section 8, where we prove that a more general version of Theorem 4 holds even when the roots $r_{i}$ are not distinct. 
3.5. Computational issues. We have seen in the above theorems and corollaries that we can decide the uniqueness of representations of certain sums in closed form if we can decide whether or not the $N=\left(\begin{array}{c}p+d-1 \\ p\end{array}\right)$ formally distinct monomials of degree $p$ in the roots $r_{1}, \ldots, r_{d}$ are actually all different, when evaluated as complex numbers. We note here that there are various ways in which this can be done without computing the roots.

For example, the elementary symmetric functions of these $N$ monomials in the $r_{i}$ 's are symmetric functions in the $r_{i}$ 's themselves. Since any symmetric function of the roots of a polynomial can be computed rationally in terms of its coefficients, the same applies to these. Once the elementary symmetric functions of the $N$ monomials of degree $p$ have been computed, the discriminant of the polynomial whose coefficients they are can be computed in the usual way. Thus, our condition on the roots of $F$ can be tested without finding the roots. It would be interesting to investigate in general this "hyperdiscriminant" of degree $p$ that is attached to a polynomial $f$, particularly with regard to how it factors when expressed in terms of the coefficients of $f$.

\section{Uniqueness and Dimension: FibonacCi POWER SUmS}

In the next two sections, we investigate the uniqueness of the expansions guaranteed by Theorems 1 and 2 . Motivated by the example in 3.3, we first consider this question for expansions of the form

$$
\sum_{j=0}^{p} \Lambda_{p, j} F(n)^{j} F(n+1)^{p-j}
$$

and, more generally,

$$
\sum_{j=0}^{p} \Lambda_{p, j} F(n)^{j} F(n+1)^{p-j}+c_{p} n+d_{p},
$$

where $F(n)$ denotes the $n$th Fibonacci number. In Section 5 we develop tools to help answer these questions for more general linear recurrences, and for other summations such as those arising in the examples in 3.1 and 3.2. The techniques in these two sections can be viewed as refinements and extensions of the ideas introduced in Section 2 to prove Theorems 1, 2, and 3.

Theorem 5. Let $\mathbf{V}=\mathbf{V}^{\infty}$ denote the vector space of complex-valued functions on $\{0,1,2, \ldots\}$, and let $\mathbf{W}_{\mathbf{p}}$ denote the subspace of $\mathbf{V}$ spanned by functions of the form $F(n)^{i} F(n+1)^{p-i}$ for $i=0, \ldots, p$, and let $\mathbf{W}_{\mathbf{p}}^{++}$denote the subspace spanned by the same monomial expressions together with with the functions $g(n)=n$ and $h(n)=1$. Then

(1) $\operatorname{dim}\left(\mathbf{W}_{\mathbf{p}}\right)=p+1$, and

(2) $\operatorname{dim}\left(\mathbf{W}_{\mathbf{p}}^{++}\right)= \begin{cases}p+2, & \text { if } p \text { is divisible by } 4, \\ p+3, & \text { otherwise. }\end{cases}$

Corollary 6. The functions $F(n)^{i} F(n+1)^{p-i}, 1 \leq i \leq p$, are linearly independent, and the set

$$
\left\{F(n)^{i} F(n+1)^{p-i}\right\}_{1 \leq i \leq p} \cup\{n, 1\}
$$

is linearly independent unless $p$ is divisible by 4 , in which case there is a single relation among its elements. 
Proof. Let $r_{1}=(1+\sqrt{5}) / 2$ and $r_{2}=(1-\sqrt{5}) / 2$ denote the roots of the Fibonacci recurrence polynomial. As noted earlier in the proof of Theorem $1, r_{1}^{n}$ and $r_{2}^{n}$ may be expressed as linear combinations of $F(n)$ and $F(n+1)$ and vice versa. Consequently, $\mathbf{W}_{\mathbf{p}}$ is the linear span of $r_{1}^{n i} r_{2}^{n(p-i)}, i=0, \ldots, p$, and to prove statement (1) it suffices to show that these functions are linearly independent. But this follows immediately from the fact that the numbers $r_{1}^{i} r_{2}^{(p-i)}$ are distinct, for $i=0, \ldots, p$.

To prove part (2), consider the $(p+3) \times(p+3)$ matrix $M_{p}$ whose $i$ th column is equal to the vector $\left(1, \theta_{i}, \theta_{i}^{2}, \ldots, \theta_{i}^{p+2}\right)$, where $\theta_{i}=r_{1}^{i} r_{2}^{(p-i)}, i=0, \ldots, p$, and whose last two columns are the vectors $(1,1, \ldots, 1)$ and $(0,1, \ldots, p+2)$. For example, when $p=2$ we have

$$
M_{2}=\left(\begin{array}{ccccc}
1 & 1 & 1 & 1 & 0 \\
r_{2}^{2} & r_{1} r_{2} & r_{1}^{2} & 1 & 1 \\
r_{2}^{4} & r_{1}^{2} r_{2}^{2} & r_{1}^{4} & 1 & 2 \\
r_{2}^{6} & r_{1}^{3} r_{2}^{3} & r_{1}^{6} & 1 & 3 \\
r_{2}^{8} & r_{1}^{4} r_{2}^{4} & r_{1}^{8} & 1 & 4
\end{array}\right)
$$

Note that $\operatorname{det} M_{p}$ is the derivative at $t=1$ of the $(p+3) \times(p+3)$ Vandermonde determinant $\operatorname{det} M_{p}(t)$, where $M_{p}(t)$ is the matrix whose first $p+2$ columns are the same as those of $M_{p}$, and whose last column is $\left(1, t, t^{2}, \ldots, t^{p+2}\right)$. We have

$$
\begin{aligned}
\operatorname{det} M_{p} & =\left.\frac{d}{d t} \operatorname{det} M_{p}(t)\right|_{t=1} \\
& =\left.\frac{d}{d t}\left(\prod_{0 \leq i<j \leq p}\left(\theta_{j}-\theta_{i}\right) \prod_{0 \leq i \leq p}\left(1-\theta_{i}\right) \prod_{0 \leq i \leq p}\left(t-\theta_{i}\right)(t-1)\right)\right|_{t=1} \\
& =\prod_{0 \leq i<j \leq p}\left(\theta_{j}-\theta_{i}\right) \prod_{0 \leq i \leq p}\left(1-\theta_{i}\right)^{2} .
\end{aligned}
$$

It follows that $\operatorname{det} M_{p}=0$ only when $t=1$ is a multiple root of $\operatorname{det} M_{p}(t)$, i.e., $r_{1}^{i} r_{2}^{p-i}=1$ for some $i$. Using the fact that $r_{1} r_{2}=-1$, it is easy to show that this property holds if and only if $p$ is a multiple of 4 . Thus, when $p$ is not a multiple of 4 , the columns of $M_{p}$ are linearly independent and we have $\operatorname{dim}\left(\mathbf{W}_{\mathbf{p}}^{++}\right)=p+3$.

If $p$ is a multiple of 4 , then $M_{p}$ contains exactly two columns of $1 \mathrm{~s}$. If one of these columns is suppressed, the argument just given shows that the remaining columns are linearly independent. Hence $\operatorname{rank}\left(M_{p}\right)=p+2$ and $\operatorname{dim}\left(\mathbf{W}_{\mathbf{p}}^{++}\right) \geq p+2$. Since the dimension is clearly at most $p+2$ in this case, the theorem is proved.

\section{UNiqUENESS AND DIMENSION: OTHER RECURRENCES WITH DISTINCT ROOTS}

Analogs of Theorem 5 hold for more general recurrences with distinct roots, but the exact statements depend on properties of the associated roots. The following theorem concerns relations among monomials of type (4), and allows precise dimension computations in many cases.

Theorem 7. Let $F(n)$ be a solution to a linear recurrence of order d whose associated roots $r_{1}, r_{2}, \ldots, r_{d}$ are distinct, and let $p$ and $q$ be distinct positive integers. Let $\mathbf{W}_{\mathbf{p}}$ denote the subspace of $\mathbf{V}=\mathbf{V}^{\infty}$ spanned by all degree $p$ monomials of the form

$$
F(n)^{i_{1}} F(n+1)^{i_{2}} \cdots F(n+d-1)^{i_{d}},
$$


where $i_{1}+i_{2}+\cdots+i_{d}=p$ and $i_{j} \geq 0$ for all $j$. Let $\mathbf{W}_{\mathbf{q}}^{+}$denote the subspace spanned by all degree $q$ monomials

$$
\begin{aligned}
& F(n)^{i_{1}} F(n+1)^{i_{2}} \cdots F(n+d-1)^{i_{d}} \quad \text { and } \\
n F(n)^{i_{1}} F(n+1)^{i_{2}} \cdots F(n+d-1)^{i_{d}}, &
\end{aligned}
$$

where $i_{1}+i_{2}+\cdots i_{d}=q$ and $i_{j} \geq 0$ for all $j$. And, finally, let $\mathbf{W}_{\mathbf{p}, \mathbf{q}}^{++}=\mathbf{W}_{\mathbf{p}}+\mathbf{W}_{\mathbf{q}}^{+}$ denote the subspace spanned by all of the above monomials. Then

$\operatorname{dim}\left(\mathbf{W}_{\mathbf{p}}\right)=\left|S_{p}\right|, \quad \operatorname{dim}\left(\mathbf{W}_{\mathbf{q}}^{+}\right)=2\left|S_{q}\right|, \quad$ and $\quad \operatorname{dim}\left(\mathbf{W}_{\mathbf{p}, \mathbf{q}}^{++}\right)=\left|S_{p}\right|+2\left|S_{q}\right|-\left|S_{p} \cap S_{q}\right|$, where $S_{p}=\left\{r_{1}^{i_{1}} r_{2}^{i_{2}} \cdots r_{d}^{i_{d}} \mid i_{1}+i_{2}+\cdots+i_{d}=p\right\}$ and $S_{q}=\left\{r_{1}^{i_{1}} r_{2}^{i_{2}} \cdots r_{d}^{i_{d}} \mid i_{1}+i_{2}+\right.$ $\left.\cdots+i_{d}=q\right\}$ are the sets of monomials in the $r_{i}$ of degrees $p$ and $q$, respectively, both viewed as subsets of the complex numbers.

Corollary 8. The sets of monomials generating $\mathbf{W}_{\mathbf{p}}, \mathbf{W}_{\mathbf{q}}^{+}$, and $\mathbf{W}_{\mathbf{p}, \mathbf{q}}^{++}$, respectively, are linearly independent if and only if evaluations of formally distinct monomials in the sets $S_{p}, S_{q}$ and $S_{p} \cup S_{q}$ yield distinct complex numbers.

The proof of Theorem 7 is analogous to that given for Theorem 5 , but more careful analysis is required. First consider the case of $\mathbf{W}_{\mathbf{p}}$. As noted in Section 2, each of the functions $F(n), F(n+1), \ldots, F(n+d-1)$ lies in the linear span of $r_{1}^{n}, r_{2}^{n}, \ldots, r_{d}^{n}$, and conversely. Hence $\mathbf{W}_{\mathbf{p}}$ is spanned by the set $\left\{\theta_{1}^{n}, \theta_{2}^{n}, \ldots, \theta_{m(p, d)}^{n}\right\}$, where $m(p, d)=\left(\begin{array}{c}p+d-1 \\ p\end{array}\right)$ and the $\theta_{j}$ range over the $m(p, d)$ formally distinct monomials of degree $p$ in $r_{1}, r_{2}, \ldots, r_{d}$. Similar reasoning shows that $\mathbf{W}_{\mathbf{q}}^{+}$is spanned by the $2 m(q, d)$ functions

$$
\begin{gathered}
\psi_{1}^{n}, \psi_{2}^{n}, \ldots, \psi_{m(q, d)}^{n}, \\
n \psi_{1}^{n}, n \psi_{2}^{n}, \ldots, n \psi_{m(q, d)}^{n},
\end{gathered}
$$

where the $\psi_{j}$ range over all formally distinct monomials of degree $q$ in $r_{1}, \ldots, r_{d}$, and finally, $\mathbf{W}_{\mathbf{p}, \mathbf{q}}^{++}$is spanned by the $m(p, d)+2 m(q, d)$ functions

$$
\begin{gathered}
\theta_{1}^{n}, \theta_{2}^{n}, \ldots, \theta_{m(p, d)}^{n}, \\
\psi_{1}^{n}, \psi_{2}^{n}, \ldots, \psi_{m(q, d)}^{n}, \\
n \psi_{1}^{n}, n \psi_{2}^{n}, \ldots, n \psi_{m(q, d)}^{n},
\end{gathered}
$$

where $\theta_{i}$ and $\psi_{j}$ are defined as above. Theorem 7 is now an immediate consequence of the following lemma.

Lemma 9. Let $\omega_{1}, \omega_{2}, \ldots, \omega_{m}$ be complex numbers, and let $a_{1}, a_{2}, \ldots, a_{m}$ be positive integers. Then the functions

$$
n^{j} \omega_{i}^{n}, \quad 1 \leq i \leq m, 0 \leq j \leq a_{i}-1,
$$

are linearly independent if and only if the $\omega_{i}$ are distinct.

Lemma 9 is a standard component of the classical theory of finite difference equations (e.g., [3], Chapter 11), indeed it is the justification for the usual method of solution of such equations. It is easy to give a direct proof via generating functions, or, alternatively, one can give a Vandermonde-type proof based on the following elegant determinant formula (2], but also see [6] for an extensive history of this formula). 
Theorem 10. Let $x_{1}, x_{2}, \ldots, x_{n}$ be indeterminates, and let $a_{1}, a_{2}, \ldots, a_{n}$ be positive integers with $\sum_{i} a_{i}=N$. For all $t$, and for any integer $k \geq 1$, let

$$
\rho_{N}(t, k)=\frac{d^{k}}{d t^{k}}\left(1, t, t^{2}, \ldots, t^{N-1}\right) .
$$

Let $M\left(a_{1}, a_{2}, \ldots, a_{n}\right)$ be the $N \times N$ matrix whose first $a_{1}$ rows are $\rho_{N}\left(x_{1}, 0\right), \ldots$, $\rho_{N}\left(x_{1}, a_{1}-1\right)$, and whose next $a_{2}$ rows are $\rho_{N}\left(x_{2}, 0\right), \ldots, \rho_{N}\left(x_{2}, a_{2}-1\right)$, and so forth. Then

$$
\operatorname{det} M\left(a_{1}, \ldots, a_{n}\right)=\prod_{i=1}^{n}\left(a_{i}-1\right) ! ! ! \prod_{1 \leq i<j \leq n}\left(x_{j}-x_{i}\right)^{a_{i} a_{j}}
$$

where $k ! ! !$ denotes $1 ! 2 ! \cdots k$ ! and $0 ! ! !=1$.

For example,

$$
M(1,2,3)=\left(\begin{array}{cccccc}
1 & x_{1} & x_{1}^{2} & x_{1}^{3} & x_{1}^{4} & x_{1}^{5} \\
1 & x_{2} & x_{2}^{2} & x_{2}^{3} & x_{2}^{4} & x_{2}^{5} \\
0 & 1 & 2 x_{2} & 3 x_{2}^{2} & 4 x_{2}^{3} & 5 x_{2}^{4} \\
1 & x_{3} & x_{3}^{2} & x_{3}^{3} & x_{3}^{4} & x_{3}^{5} \\
0 & 1 & 2 x_{3} & 3 x_{3}^{2} & 4 x_{3}^{3} & 5 x_{3}^{4} \\
0 & 0 & 2 & 6 x_{3} & 12 x_{3}^{2} & 20 x_{3}^{3}
\end{array}\right)
$$

and

$$
\operatorname{det} M(1,2,3)=2\left(x_{2}-x_{1}\right)^{2}\left(x_{3}-x_{1}\right)^{3}\left(x_{3}-x_{2}\right)^{6} .
$$

Theorem 7 describes relations among closed form expressions of type (4), but the proof also yields similar results for expressions of type (3).

Corollary 11. Let $F(n)$ be a solution to a linear recurrence of order d whose associated roots are distinct. Let $\mathbf{W}_{\mathbf{d}}^{*}$ denote the space spanned by monomial functions of type (3). Then $\operatorname{dim} \mathbf{W}_{\mathbf{d}}^{*}=|S|+2|T|$, where $S$ is the set of all monomials of the form $t_{1}^{a_{1}} t_{2}^{a_{2}} \cdots t_{k}^{a_{k}}$ and $T$ is the set of monomials of the form $t_{1}^{a_{1}+b_{1}} t_{2}^{a_{2}+b_{2}} \cdots t_{k}^{a_{k}+b_{k}}$ and, for each $i, t_{i}$ is one of the roots $r_{1}, r_{2}, \ldots, r_{d}$. The monomial functions of type (3) are linearly independent if and only if formally distinct monomials in $S \cup T$ correspond to distinct complex numbers.

We omit the proof, which is analogous to that of the proof of Theorem 7. We note that the set $S \cup T$ in Corollary 11] is a subset of the set $S_{p} \cup S_{q}$ appearing in Corollary 8 , and thus we obtain the following result.

Corollary 12. Under the assumptions of Corollary 11, if the monomial functions of type (4) are linearly independent, then so are the monomial functions of type (3).

We will now apply these results to some of the formulæ in Sections 3.1 and 3.2.

Corollary 13. For the Fibonacci sum $f(n)$ appearing in (10), equation (11) gives the unique F-closed formula of type (3) and (12) gives the unique F-closed formula of type (4).

Proof. By Theorem 7 and Corollary 12, we need only check that if $r_{1}$ and $r_{2}$ denote the roots of the Fibonacci recurrence, then

$$
r_{1}^{2}, r_{1} r_{2}, r_{2}^{2}, r_{1}^{3}, r_{1}^{2} r_{2}, r_{1} r_{2}^{2}, \text { and } r_{2}^{3}
$$

are distinct real numbers. This is an elementary calculation. 
Corollary 14. For the sum $g(n)=\sum_{j=0}^{n-1} G(j)^{2}$ arising in the subword avoidance problem with $A=2$, solutions $g(n)$ of type (4) are all given by (15) plus constant multiples of relation (16).

Proof. The roots of the recurrence equation $t^{3}-2 t^{2}+1=0$ are $r_{1}, r_{2}, r_{3}$, where $r_{1}$ and $r_{2}$ are roots of the Fibonacci recurrence and $r_{3}=1$. By Theorem 7 , the dimension of the space $\mathbf{W}_{\mathbf{2}, \mathbf{0}}^{++}$spanned by the six degree- 2 monomials in $G(n)$, $G(n+1)$ and $G(n+2)$ together with 1 and $n$ is equal to $\left|S_{2}\right|+2\left|S_{0}\right|-\left|S_{2} \cap S_{0}\right|$, where $S_{2}=\left\{r_{1}^{2}, r_{2}^{2}, 1, r_{1} r_{2}, r_{1}, r_{2}\right\}$, and $S_{0}=\{1\}$. Elementary calculation shows that this dimension is equal to 7 , hence the monomials generating $\mathbf{W}_{\mathbf{2}, \mathbf{0}}^{++}$are linearly independent apart from a one-parameter family of relations.

Next we consider the case $A=3$, as a warmup for the general case $A>2$.

Corollary 15. For the sum $g(n)=\sum_{j=0}^{n-1} G(j)^{2}$ arising in the subword avoidance problem with $A=3$, formula (14) gives the unique $G$-closed formula of type (3).

Proof. Here the recurrence equation is $t^{3}-3 t^{2}+1=0$, which has roots $r_{1}=$ $1+\eta+\eta^{17}, r_{2}=1+\eta^{7}+\eta^{11}, r_{3}=1+\eta^{5}+\eta^{13}$, where $\eta=e^{2 \pi i / 18}$ is an 18 th root of unity. Again, by Theorem[ 7 the dimension of the space of monomials $\mathbf{W}_{\mathbf{2}, \mathbf{0}}^{++}$is equal to $\left|S_{2}\right|+2\left|S_{0}\right|-\left|S_{2} \cap S_{0}\right|$, where $S_{2}=\left\{r_{1}^{2}, r_{2}^{2}, r_{3}^{2}, r_{1} r_{2}, r_{1} r_{3}, r_{2} r_{3}\right\}$, and $S_{0}=\{1\}$. A slightly less elementary calculation shows that the formal monomials in $S_{2} \cup S_{0}$ are distinct, so that $\operatorname{dim}\left(\mathbf{W}_{\mathbf{2}, \mathbf{0}}^{++}\right)=8$ and the monomial functions generating $\mathbf{W}_{\mathbf{2}, \mathbf{0}}^{++}$ are linearly independent.

Corollary 16. For the more general power sum $g(n)=\sum_{j=0}^{n-1} G(j)^{p}$ arising in the subword avoidance problem, with $p>0$ and any $A>2$, solutions of type (3) are unique if and only if $p \not \equiv 0 \bmod 6$.

Proof. An argument analogous to the calculation in Section 3.2 shows that formulæ of type (3) exist expressing $g(n)$ as linear combinations of monomials in $G(n)$, $G(n+1)$, and $G(n+2)$ of degree $p$, together with 1 and $n$. We need to compute the dimension of $\mathbf{W}_{\mathbf{p}, \mathbf{0}}^{++}$, which by Theorem 7 is equal to $\left|S_{p}\right|+2\left|S_{0}\right|-\left|S_{p} \cap S_{0}\right|$, where $S_{0}=\{1\}$ and $S_{p}$ is the set of all degree- $p$ monomials in $r_{1}, r_{2}$, and $r_{3}$, where $r_{1}, r_{2}$, and $r_{3}$ are roots of the recurrence equation $t^{3}-A t^{2}+1=0$.

If $p$ is not divisible by 6 , the proof will be complete if we can show that formally distinct monomials in $S_{p}$ evaluate to distinct complex (actually real) numbers, and none of them equals 1 . Suppose that $r_{1}^{e_{1}} r_{2}^{e_{2}} r_{3}^{e_{3}}=r_{1}^{f_{1}} r_{2}^{f_{2}} r_{3}^{f_{3}}$, where $\sum e_{i}=\sum f_{i}=p$ and $e_{i} \neq f_{i}$ for some $i$. Then by cancellation we obtain the relation $r_{i}^{u_{i}}=r_{j}^{u_{j}} r_{k}^{u_{k}}$ for some rearrangement of the indices, with $u_{i}, u_{j}, u_{k} \geq 0$ and at least one of these exponents positive. Using the relation $r_{1} r_{2} r_{3}=-1$, if necessary, to eliminate one of the roots, we obtain (after possibly reindexing) $r_{i}^{v_{i}}= \pm r_{j}^{v_{j}}$ with $v_{i}, v_{j} \geq 0$, and at least one of these exponents positive.

It is a straightforward exercise to show that the roots $r_{1}, r_{2}$ and $r_{3}$ are all real, and that, if they are arranged in decreasing order, then $r_{1}>1,0<r_{2}<1$, and $-1<r_{3}<0$. From elementary Galois theory we know that there exists an automorphism $\Phi$ of the field $K=\mathbf{Q}\left(r_{1}, r_{2}, r_{3}\right)$ such that $\Phi: r_{1} \mapsto r_{2} \mapsto r_{3} \mapsto r_{1}$, i.e., it permutes the roots cyclically. Hence the equation $r_{i}^{v_{i}}= \pm r_{j}^{v_{j}}$ holds for all three cyclic permutations of the roots. At least one of these equations leads to a contradiction, since $\left|r_{1}\right|>1$ and $\left|r_{2}\right|,\left|r_{3}\right|<1$. This proves that formally distinct monomials are distinct, and it remains to show that none can equal 1. 
Suppose that $r_{1}^{e_{1}} r_{2}^{e_{2}} r_{3}^{e_{3}}=1$, and that the exponents $e_{i}$ are not all equal. Applying the identity $r_{1} r_{2} r_{3}=-1$ we obtain a relation of the form $r_{i}^{u_{i}} r_{j}^{u_{j}}= \pm 1$ for some pair of distinct $i, j$, with $u_{i}, u_{j} \geq 0$ and at least one positive. Again, this relation holds for all cyclic permutations of the indices, and consideration of absolute values leads to a contradiction in at least one case. Consequently, we must have $e_{1}=e_{2}=e_{3}=e$ for some $e$. From the relations $r_{1} r_{2} r_{3}=-1$ and $\left(r_{1} r_{2} r_{3}\right)^{e}=1$ we conclude that $e$ is even, which implies that $p$ is a multiple of 6 . This completes the proof that monomials in the $G$ are linearly independent when $p \not \equiv 0 \bmod 6$. When $p=6 \mathrm{~m}$ the relation $\left(r_{1} r_{2} r_{3}\right)^{2 m}=1$ gives relations in the $G$ of degree 6 , and so the proof of Corollary 16 is complete.

\section{The General CASE of Multiple Roots}

In this section we show the result of dropping the assumption of distinct roots. We also consider a somewhat more general summation problem, viz.

$$
f(n)=\sum_{j=0}^{n-1} F_{1}\left(a_{1} n+b_{1} j+c_{1}\right) F_{2}\left(a_{2} n+b_{2} j+c_{2}\right) \cdots F_{k}\left(a_{k} n+b_{k} j+c_{k}\right),
$$

in which the factors of the summand may be different $C$-finite functions. The analysis in this general case is similar to that in the case of distinct roots, but some additional machinery is required. The main result is the following, which is a generalization and also a strengthening of Theorem 1 .

Theorem 17. Let $F_{1}, F_{2}, \ldots, F_{k}$ be given $C$-finite sequences. Suppose that, for each $i=1, \ldots, k, F_{i}(n)$ satisfies a recurrence of minimal degree $D(i)$ whose polynomial equation has $d(i)$ distinct roots. Denote these roots by $r_{1}^{(i)}, \ldots, r_{d(i)}^{(i)}$, and let $e_{1}^{(i)}, \ldots, e_{d(i)}^{(i)}$ be their respective multiplicities, so that $D(i)=\sum_{j} e_{j}^{(i)}$. Finally, let $\Delta(i)=\max _{1 \leq j \leq d(i)}\left(e_{j}^{(i)}-1\right)$. Then the sum $f(n)$, of (23), can be expressed as a linear combination of the monomials

$$
F_{1}\left(\left(a_{1}+b_{1}\right) n+i_{1}\right) \ldots F_{k}\left(\left(a_{k}+b_{k}\right) n+i_{k}\right) \quad\left(0 \leq i_{\nu} \leq D(\nu)-1 ; 1 \leq \nu \leq k\right),
$$

and

$$
\psi_{i_{1}, \ldots, i_{k}}(n) F_{1}\left(a_{1} n+i_{1}\right) \ldots F_{k}\left(a_{k} n+i_{k}\right) \quad\left(0 \leq i_{\nu} \leq D(\nu)-1 ; 1 \leq \nu \leq k\right),
$$

in which $\psi_{i_{1}, \ldots, i_{k}}(n)$ is a polynomial whose degree is bounded above by

- zero, i.e., the factor $\psi$ can be omitted, if for all sequences $\left(m_{1}, m_{2}, \ldots, m_{k}\right)$ with $1 \leq m_{i} \leq d(i)$, we have $\prod_{i=1}^{k}\left(r_{m_{i}}^{(i)}\right)^{b_{i}} \neq 1$, and

- $1+\sum_{i}\left\{\Delta(i): a_{i}=0\right\}$ otherwise.

Proof. We have, for the sum $f(n)$ of (23),

$$
f(n)=\sum_{j=0}^{n-1} \prod_{\ell=1}^{k} \sum_{m=1}^{d(\ell)} \sum_{h=0}^{e_{m}^{(\ell)}-1} \lambda_{m, h}^{(\ell)}\left(a_{\ell} n+b_{\ell} j+c_{\ell}\right)^{h}\left(r_{m}^{(\ell)}\right)^{a_{\ell} n+b_{\ell} j+c_{\ell}},
$$

where the coefficients $\lambda_{m, h}^{(\ell)}$ are defined by the form of the $F_{i}$ 's, namely

$$
F_{i}(n)=\sum_{m=1}^{d(i)} \sum_{h=0}^{e_{m}^{(i)}-1} \lambda_{m, h}^{(i)} n^{h}\left(r_{m}^{(i)}\right)^{n}
$$


If we expand the product and the two inner sums in (26), we find that a typical term is of the form

$$
K\left(a_{1} n+b_{1} j+c_{1}\right)^{h_{1}}\left(r_{m_{1}}^{(1)}\right)^{a_{1} n+b_{1} j+c_{1}} \ldots\left(a_{k} n+b_{k} j+c_{k}\right)^{h_{k}}\left(r_{m_{k}}^{(k)}\right)^{a_{k} n+b_{k} j+c_{k}},
$$

where $0 \leq h_{i} \leq e_{m_{i}}^{(i)}-1$ for $1 \leq i \leq k$. If we write

$$
\left(a_{i} n+b_{i} j+c_{i}\right)^{h_{i}}=\left(a_{i}(n-j)+\left(a_{i}+b_{i}\right) j+c_{i}\right)^{h_{i}}
$$

and further expand each of these factors, we find that our typical term can now be expressed as

$$
K j^{q}(n-j)^{r}\left(\left(r_{m_{1}}^{(1)}\right)^{b_{1}} \ldots\left(r_{m_{k}}^{(k)}\right)^{b_{k}}\right)^{j}\left(\left(r_{m_{1}}^{(1)}\right)^{a_{1}} \ldots\left(r_{m_{k}}^{(k)}\right)^{a_{k}}\right)^{n}
$$

in which

$q \leq \sum\left\{h_{i}: a_{i}+b_{i} \neq 0\right\}, \quad r \leq \sum\left\{h_{i}: a_{i} \neq 0\right\}$, and $q+r \leq \sum_{i} h_{i} \leq \sum_{i}\left(e_{m_{i}}^{(i)}-1\right)$.

At this point we need the following result.

Lemma 18. We have

$$
\sum_{j=0}^{n-1} j^{a}(n-j)^{b} x^{j}= \begin{cases}P_{a}(n) x^{n}+P_{b}(n), & \text { if } x \neq 1, \\ P_{a+b+1}(n), & \text { if } x=1,\end{cases}
$$

where $P_{s}(n)$ denotes a generic polynomial of degree $s$, whose coefficients may depend on $x$.

Proof. Suppose that $x \neq 1$. If $b=0$, we have

$$
\sum_{j=0}^{n-1} j^{a} x^{j}=\left(x \frac{d}{d x}\right)^{a} \sum_{j=0}^{n-1} x^{j}=\left(x \frac{d}{d x}\right)^{a}\left(\frac{x^{n}-1}{x-1}\right),
$$

which is of the form stated when $b=0$. For $b>0$ we have

$$
\begin{aligned}
& \sum_{j=0}^{n-1} j^{a}(n-j)^{b} x^{j} \\
& \quad=\left(x \frac{d}{d x}\right)^{a} \sum_{j=0}^{n-1}(n-j)^{b} x^{j}=\left(x \frac{d}{d x}\right)^{a} \sum_{j=1}^{n} j^{b} x^{n-j}=\left(x \frac{d}{d x}\right)^{a} x^{n} \sum_{j=1}^{n} j^{b} x^{-j} \\
& \quad=\left(x \frac{d}{d x}\right)^{a} x^{n}\left(\left(P_{b}(n)+n^{b}\right) x^{-n}+K\right)=\left(x \frac{d}{d x}\right)^{a}\left(P_{b}(n)+n^{b}+K x^{n}\right)
\end{aligned}
$$

which is evidently of the desired form. The case $x=1$ is elementary. 
If we sum the typical term (27) over $j=0, \ldots, n-1$ and use the lemma, we find that the overall sum $f(n)$ is a sum of expressions of the form

$$
\begin{aligned}
& \sum_{j=0}^{n-1} K j^{q}(n-j)^{r}\left(\left(r_{m_{1}}^{(1)}\right)^{b_{1}} \ldots\left(r_{m_{k}}^{(k)}\right)^{b_{k}}\right)^{j}\left(\left(r_{m_{1}}^{(1)}\right)^{a_{1}} \ldots\left(r_{m_{k}}^{(k)}\right)^{a_{k}}\right)^{n} \\
& =K\left(\left(r_{m_{1}}^{(1)}\right)^{a_{1}} \ldots\left(r_{m_{k}}^{(k)}\right)^{a_{k}}\right)^{n} \\
& \times \begin{cases}P_{q}(n)\left(\left(r_{m_{1}}^{(1)}\right)^{b_{1}} \ldots\left(r_{m_{k}}^{(k)}\right)^{b_{k}}\right)^{n}+P_{r}(n), & \text { if } \Theta \neq 1, \\
P_{q+r+1}(n), & \text { if } \Theta=1 .\end{cases} \\
& =\left\{\begin{aligned}
K P_{q}(n)\left(\left(r_{m_{1}}^{(1)}\right)^{a_{1}+b_{1}} \ldots\left(r_{m_{k}}^{(k)}\right)^{a_{k}+b_{k}}\right)^{n} & \\
\quad+K P_{r}(n)\left(\left(r_{m_{1}}^{(1)}\right)^{a_{1}} \ldots\left(r_{m_{k}}^{(k)}\right)^{a_{k}}\right)^{n}, & \text { if } \Theta \neq 1, \\
K P_{q+r+1}(n)\left(\left(r_{m_{1}}^{(1)}\right)^{a_{1}} \ldots\left(r_{m_{k}}^{(k)}\right)^{a_{k}}\right)^{n}, & \text { if } \Theta=1,
\end{aligned}\right.
\end{aligned}
$$

where $\Theta=\left(r_{m_{1}}^{(1)}\right)^{b_{1}} \ldots\left(r_{m_{k}}^{(k)}\right)^{b_{k}}, q$ and $r$ satisfy the bounds given in (28), and $P_{s}(n)$ denotes a generic polynomial of degree $s$.

Considering each of the three terms appearing in the last member of (29), we first have

$$
\begin{aligned}
K P_{q}(n)\left(\left(r_{m_{1}}^{(1)}\right)^{a_{1}+b_{1}} \ldots\left(r_{m_{k}}^{(k)}\right)^{a_{k}+b_{k}}\right)^{n} & =K P_{q}(n) \prod_{\left\{i: a_{i}+b_{i} \neq 0\right\}}\left(r_{m_{i}}^{(i)}\right)^{\left(a_{i}+b_{i}\right) n} \\
& =\left(\sum_{j=0}^{q} \beta_{j} n^{j}\right) \prod_{\left\{i: a_{i}+b_{i} \neq 0\right\}}\left(r_{m_{i}}^{(i)}\right)^{\left(a_{i}+b_{i}\right) n},
\end{aligned}
$$

say. Since $q \leq \sum_{i}\left\{h_{i}: a_{i}+b_{i} \neq 0\right\} \leq \sum_{i}\left\{e_{m_{i}}^{(i)}-1: a_{i}+b_{i} \neq 0\right\}$, each exponent $j$ in the range $0 \leq j \leq q$ can be written (in many ways) as $j=j_{1}+j_{2}+\cdots+j_{k}$, where $0 \leq j_{i} \leq e_{m_{i}}^{(i)}-1$ and $j_{i}=0$ if $a_{i}+b_{i}=0$. Hence the last member above may be expressed as

$$
\sum_{j=0}^{q} \beta_{j} \prod_{\left\{i: a_{i}+b_{i} \neq 0\right\}} n^{j_{i}}\left(r_{m_{i}}^{(i)}\right)^{n\left(a_{i}+b_{i}\right)} \quad\left(0 \leq j_{i} \leq e_{m_{i}}^{(i)}\right)
$$

Now we observe that the solution space of the recurrence satisfied by $F_{i}(n)$ has dimension $D(i)$, and that the $D(i)$ shifted sequences

$$
\left\{F_{i}(n)\right\},\left\{F_{i}(n+1)\right\}, \ldots,\left\{F_{i}(n+D(i)-1)\right\}
$$

are linearly independent, since in the contrary case the function $F_{i}$ would satisfy a recurrence of degree $<D(i)$. Consequently these $D(i)$ sequences are a basis for the solution space, and therefore each of the functions $n^{j}\left(r_{m}^{(i)}\right)^{n}, j=0,1, \ldots, e_{m}^{(i)}-$ $1, m=1, \ldots, d(i)$ can be written as a linear combination of $F_{i}(n), F_{i}(n+1), \ldots$, $F_{i}(n+D(i)-1)$.

Thus we return to our general term (30), and we replace each of the monomials of the form $n^{j} r_{m}^{n(a+b)}$ by such a linear combination of functions of the form

$$
F((a+b) n), F((a+b) n+1), \ldots, F((a+b) n+D(i)-1),
$$


and expand everything again. Now our general term is of the form (24) in the statement of the theorem, which concludes the treatment of the first term in the final display of (29). The second term,

$$
K P_{r}(n)\left(\left(r_{m_{1}}^{(1)}\right)^{a_{1}} \ldots\left(r_{m_{k}}^{(k)}\right)^{a_{k}}\right)^{n},
$$

may be handled similarly. In the third case, which occurs when $\Theta=1$, powers of $n$ in $P_{q+r+1}(n)$ can be redistributed as in (30) provided that each corresponding $a_{i}$ is nonzero. If all $a_{i} \neq 0$, a term of degree at most 1 remains, since $q+r \leq \sum_{i} e_{m_{i}}^{(i)}-1$; more generally, the residual exponent is at most equal to $1+\sum_{i}\left\{\Delta(i): a_{i}=0\right\}$.

By keeping track of the number of terms of the form $n^{j} \prod r_{m}^{a n}$ and $n^{j} \prod r_{m}^{(a+b) n}$ being summed in (29) we can give a bound on the $C$-finite degree of $f(n)$, generalizing Theorem 3 to the case of multiple roots and different $F_{i}$.

Corollary 19. Suppose that $F_{1}, F_{2}, \ldots, F_{k}$ are as defined in Theorem 17. Let $M$ denote the number of algebraically distinct monomials generated by (24) and (25), as defined in the paragraph preceding Theorem 3, Then the sum $f(n)$ in (23) is $C$ finite, of degree at most $M$. The coefficients of the monomials expressing $f(n)$ as a linear combination of those monomials can be found by solving equations involving at most $M$ values of $f(n)$. If a solution is valid for the first $M$ values of $n$, then it is valid for all values of $n$.

Proof. Assume first that no expression of the form $\Theta=\prod_{\ell=1}^{k}\left(r_{m_{\ell}}^{(\ell)}\right)^{b_{\ell}}$ equals 1 . Define $Q=\left\{\ell \mid a_{\ell}+b_{\ell} \neq 0\right\}$ and $R=\left\{\ell \mid a_{\ell} \neq 0\right\}$. The proof of Theorem 17 shows that $f(n)$ is in the linear span of the set $U_{Q} \cup U_{R}$, where $U_{Q}$ is the set of functions of the form

$$
\prod_{\ell \in Q} n^{j_{\ell}}\left(r_{m_{\ell}}^{(\ell)}\right)^{\left(a_{\ell}+b_{\ell}\right) n}
$$

where $1 \leq m_{\ell} \leq d(\ell)$ and $0 \leq j_{\ell} \leq e_{m_{\ell}}^{(\ell)}-1$, and $U_{R}$ is the set of functions of the form

$$
\prod_{\ell \in R} n^{j_{\ell}}\left(r_{m_{\ell}}^{(\ell)}\right)^{a_{\ell} n}
$$

where $1 \leq m_{\ell} \leq d(\ell)$ and $0 \leq j_{\ell} \leq e_{m_{\ell}}^{(\ell)}-1$. We claim that $\left|U_{Q} \cup U_{R}\right| \leq M$. The argument is similar to the one used in Section 2 to prove Theorem 3 in the distinct root case: if $\theta \neq 0$, we define a map $F_{\ell}(\theta n+i) \mapsto n^{j}\left(r_{m}^{(\ell)}\right)^{\theta n}$, where $i \mapsto(j, m)$ is some enumeration of the $D(\ell)$ pairs with $1 \leq m \leq d(\ell), 0 \leq j \leq e_{m_{\ell}}^{(\ell)}-1$. This map extends to a well-defined surjective map from the set of equivalence classes of monomials of type (24) and (25) to the set $U_{Q} \cup U_{R}$. The maximum $C$-finite degree of any function in $\left\langle U_{Q} \cup U_{R}\right\rangle$ is equal to the dimension of that space, and hence the $C$-finite degree of $f(n)$ is less than or equal to $M$.

Next suppose that there exist expressions of the form $\Theta=\prod_{\ell=1}^{k}\left(r_{m_{\ell}}^{(\ell)}\right)^{b_{\ell}}$ equal to 1. The proof of Theorem 17 shows that $f(n)$ is in the linear span of the set $U_{Q} \cup W_{R}$, where $U_{Q}$ is as defined above, and $W_{R}$ is the set of functions of the form

$$
n^{h} \prod_{\ell \in R}\left(r_{m_{\ell}}^{(\ell)}\right)^{a_{\ell} n}
$$


where $1 \leq m_{\ell} \leq d(\ell)$ and $0 \leq h \leq H$, where

$$
H=1+\sum_{\ell \in R}\left(e_{m_{\ell}}^{(\ell)}-1\right)+\sum_{\ell \notin R}\left(e_{m_{\ell}^{*}}^{(\ell)}-1\right)
$$

and, for a given sequence $m_{\ell}$ with $\ell \in R$, the $m_{\ell}^{*}$ are chosen to maximize $H$ over all sequences $\left(m_{1}, \ldots, m_{k}\right)$ such that $\prod_{\ell \in R}\left(r_{m_{\ell}}^{(\ell)}\right)^{b_{\ell}} \prod_{\ell \notin R}\left(r_{m_{\ell}^{*}}^{(\ell)}\right)^{b_{\ell}}=1$.

As in the first case, the map $F_{\ell}(\theta n+i) \mapsto n^{j}\left(r_{m}^{\ell}\right)^{\theta n}, \theta \neq 0$, extends to a welldefined surjective map from the set of equivalence classes of type (24) and (25) to the set $U_{Q} \cup W_{R}$, proving that $\left|U_{Q} \cup W_{R}\right| \leq M$. Hence the $C$-finite degree of $f(n)$ is at most $M$ in all cases, and the remaining assertions follow immmediately.

It is possible to give another bound on the $C$-finite degree that is sometimes sharper than the one in Corollary 19.

Corollary 20. Suppose that $F_{1}, F_{2}, \ldots, F_{k}$ are as in Theorem 17, where each $F_{i}$ is $C$-finite of degree $D(i)$, with $d(i)$ distinct roots. Then the $C$-finite degree of $f(n)$ is bounded by

$$
\left(\prod_{\ell \in Q} d(\ell)\right)\left(1+\sum_{\ell \in Q}\left(\frac{D(\ell)}{d(\ell)}-1\right)\right)+\left(\prod_{\ell \in R} d(\ell)\right)\left(1+\sum_{\ell \in R}\left(\frac{D(\ell)}{d(\ell)}-1\right)\right)
$$

where $Q=\left\{\ell \mid a_{\ell}+b_{\ell} \neq 0\right\}$ and $R=\left\{\ell \mid a_{\ell} \neq 0\right\}$. This last expression is in turn bounded by

$$
\prod_{\ell \in Q} D(\ell)+\prod_{\ell \in R} D(\ell)
$$

In all of these expressions, the empty product is taken to be equal to 1.

Proof. Again, we first consider the case where no product of the form $\Theta=$ $\prod_{\ell=1}^{k}\left(r_{m_{\ell}}^{(\ell)}\right)^{b_{\ell}}$ equals 1 . To establish the bound in (35), note that

$$
\begin{aligned}
\left|U_{Q}\right| & \leq \sum_{\left(m_{\ell}\right)_{\ell \in Q}}\left(1+\sum_{\ell \in Q}\left(e_{m_{\ell}}^{(\ell)}-1\right)\right) \\
& =\left(\prod_{\ell \in Q} d(\ell)\right)+\left(\sum_{\ell \in Q}\left(D(\ell) \prod_{j \neq \ell, j \in Q} d(j)-\prod_{\ell \in Q} d(\ell)\right)\right) \\
& =\left(\prod_{\ell \in Q} d(\ell)\right)\left(1+\sum_{\ell \in Q}\left(\frac{D(\ell)}{d(\ell)}-1\right)\right),
\end{aligned}
$$

where the first summation is over all sequences $\left(m_{\ell}\right)_{\ell \in Q}$, satisfying $1 \leq m_{\ell} \leq d(\ell)$ for $\ell \in Q$. The second term in (35) is handled similarly, and the result follows. To obtain the bound in (36), it suffices to show that

$$
\left(\prod_{\ell \in Q} d(\ell)\right)\left(1+\sum_{\ell \in Q}\left(\frac{D(\ell)}{d(\ell)}-1\right)\right) \leq \prod_{\ell \in Q} D(\ell) .
$$

To see this, divide both sides by $\prod_{\ell \in Q} d(\ell)$, and write $D(\ell) / d(\ell)=1+t_{\ell}$, where $t_{\ell} \geq 0$. It remains to show that $1+\sum_{\ell \in Q} t_{\ell} \leq \prod_{\ell \in Q}\left(1+t_{\ell}\right)$ for nonnegative $t_{\ell}$, which is obvious. This completes the proof of Corollary 19 in the first case. 
Next suppose that there are products of the form $\Theta=\prod_{\ell=1}^{k}\left(r_{m_{\ell}}^{(\ell)}\right)^{b_{\ell}}$ equal to 1 . Let $W_{R}$ be defined as in the proof of Corollary 19. We will show that $\left|U_{Q} \cup W_{R}\right|$ is bounded by (35). Arguing as in the first case, we obtain that $\left|U_{Q}\right|$ is less than or equal to the first summand in (35). Before computing the $W_{R}$ contribution, it is convenient to define

$$
W_{R}^{0}=\left\{n^{h} \prod_{\ell \in R}\left(r_{m_{\ell}}^{(\ell)}\right)^{a_{\ell} n} \mid H_{0}<h \leq H\right\},
$$

where $H_{0}=1+\sum_{\ell \in R}\left(e_{m_{\ell}}^{(\ell)}-1\right)$. We claim that

$$
\left|U_{Q} \cap W_{R}\right| \geq\left|W_{R}^{0}\right| \text {. }
$$

To see this, suppose that $n^{h} \prod_{\ell \in R}\left(r_{m_{\ell}}^{(\ell)}\right)^{a_{\ell} n} \in W_{R}^{0}$. Since $h>H_{0}$, there must exist indices $m_{\ell}^{*}$ with $\ell \notin R$ such that $\prod_{\ell \in R}\left(r_{m_{\ell}}^{(\ell)}\right)^{b_{\ell}} \prod_{\ell \notin R}\left(r_{m_{\ell}^{*}}^{(\ell)}\right)^{b_{\ell}}=1$. Assume that these have been chosen so that the right-hand side of (34) is maximized. Then

$$
n^{h-H_{0}} \prod_{\ell \in R}\left(r_{m_{\ell}}^{(\ell)}\right)^{a_{\ell} n}=n^{h-H_{0}} \prod_{\ell \in R}\left(r_{m_{\ell}}^{(\ell)}\right)^{\left(a_{\ell}+b_{\ell}\right) n} \prod_{\ell \notin R}\left(r_{m_{\ell}^{*}}^{(\ell)}\right)^{b_{\ell} n}
$$

is an element of $U_{Q} \cap W_{R}$, and it is clear that this map is injective. It follows that

$$
\begin{aligned}
\left|U_{Q} \cup W_{R}\right| & =\left|U_{Q}\right|+\left|W_{R}\right|-\left|U_{Q} \cap W_{R}\right| \\
& \leq\left|U_{Q}\right|+\left|W_{R}\right|-\left|W_{R}^{0}\right| \\
& =\left|U_{Q}\right|+\left|W_{R}-W_{R}^{0}\right| .
\end{aligned}
$$

Finally, we have

$$
\left|W_{R}-W_{R}^{0}\right| \leq \sum_{\left(m_{\ell}\right)_{\ell \in R}}\left(1+\sum_{\ell \in R}\left(e_{m_{\ell}}^{(\ell)}-1\right)\right),
$$

which is less than or equal to the second summand in (35), by the argument presented in the first case. The bound (36) follows as before, and the proof is complete.

We remark that the second case above can also be derived from the first case by a continuity argument.

\section{A STRIKING PROPERTY OF INDEFINITE SUMMATION}

When $a_{1}=a_{2}=\cdots=a_{k}=0$ in (23) we are doing indefinite summation, i.e., the problem is equivalent to finding a function $S(n)$ (called an indefinite sum function) such that

$$
S(n)-S(n-1)=F_{1}\left(b_{1} n+c_{1}\right) \cdots F_{k}\left(b_{k} n+c_{k}\right) .
$$

In this section we will show that, if no products of the form $\prod_{i=1}^{k}\left(r_{m_{i}}^{(i)}\right)^{b_{i}}$ are equal to 1 , there exists an indefinite sum function $S(n)$ expressible (formally) as a linear combination of monomials in the $F_{i}$ with coefficients that are independent of the initial conditions satisfied by the various $F_{i}$.

Theorem 4 proved this result (which we will call the independence property) for power sums $\sum_{j} F(j)^{p}$, assuming that the roots $r_{i}$ are distinct, and it is easy to extend that proof to the case of summands of the form $F_{1}\left(b_{1} n+c_{1}\right) \cdots F_{k}\left(b_{k} n+c_{k}\right)$, with different $F_{i}$, as long as the roots are distinct. Among other things, this section extends Theorem 4 to the multiple root case, where the proof turns out to be considerably more difficult. 
Similar questions have been considered in [9], where the author describes a method (different from ours) that finds indefinite sum functions in some but not all cases 1 but does not delineate these cases with a theorem. In fact, the method developed in [9] assumes the independence property; the author asserts (in somewhat vague terms, and without proof) that it holds if no products $\prod_{i}\left(r_{m_{i}}^{(i)}\right)^{b_{i}}$ are equal to 1 .

Theorem 24 (below) establishes this result, and in full generality. It dispenses with the assumption of distinct roots, and makes appropriate modifications in the case where products of the form $\prod_{i}\left(r_{m_{i}}^{(i)}\right)^{b_{i}}$ are equal to 1 . The first step in the proof is to identify a collection of functions analogous to the terms $\lambda_{m} r_{m}^{n}, 1 \leq m \leq d$, that appear in the proof of Theorem 4, which can be expressed as linear combinations of the $F(n+i)$ with coefficients that do not depend on the initial conditions.

Lemma 21. Suppose that

$$
F(n)=\sum_{m=1}^{d} \sum_{h=0}^{e_{m}-1} \lambda_{m, h} n^{h}\left(r_{m}\right)^{n},
$$

where the $r_{m}$ are distinct and nonzero, and $\lambda_{m, e_{m}-1} \neq 0$ for all $m$. For $m=1, \ldots, d$ and $h=0, \ldots, e_{m}-1$, define

$$
\Phi_{h}^{m}(n)=\sum_{i=h}^{e_{m}-1}\left(\begin{array}{l}
i \\
h
\end{array}\right) \lambda_{m, i} n^{i-h}\left(r_{m}\right)^{n} .
$$

Let $D=\sum_{m} e_{m}$. Then each of the functions $\Phi_{h}^{m}(n)$ may be expressed as a linear combination of the functions $F(n), F(n+1), \ldots, F(n+D-1)$, with coefficients that do not depend on the values of $\lambda_{m, h}$, i.e., those coefficients do not depend on the initial conditions of $F$.

Proof. Let $\mathbf{F}$ be the column vector of length $D$ whose $i$ th element is equal to $F(n+i), 0 \leq i \leq D-1$. Let $\mathbf{R}$ and $\boldsymbol{\Phi}$ be column vectors of length $D$, each indexed by $(m, h), 1 \leq m \leq d, 0 \leq h \leq e_{m}-1$, where the $(m, h)$ th element of $\mathbf{R}$ is equal to $n^{h}\left(r_{m}\right)^{n}$ and the $(m, h)$ th element of $\boldsymbol{\Phi}$ is equal to $\Phi_{e_{m-1-h}}^{m}(n)$. Then we have

$$
\mathbf{F}=\mathbf{M} \mathbf{R},
$$

where $\mathbf{M}$ is a matrix with rows indexed by $0, \ldots, D-1$ and columns indexed by $(m, h)$, whose entry in row $t$ and column $(m, h)$ is equal to

$$
\left(r_{m}\right)^{t} \sum_{i=h}^{e_{m}-1}\left(\begin{array}{l}
i \\
h
\end{array}\right) \lambda_{m, i} t^{i-h}
$$

We have the factorization

$$
\mathbf{M}=\mathbf{M}_{\mathbf{0}} \boldsymbol{\Lambda}
$$

where $\mathbf{M}_{\mathbf{0}}$ is a matrix indexed as in $\mathbf{M}$, whose entry in row $t$ and column $(m, h)$ is

$$
\left(r_{m}\right)^{t} t^{e_{m}-1-h}
$$

and $\boldsymbol{\Lambda}$ is a matrix with both rows and columns indexed by $(m, h)$, whose entry is row $(m, h)$ and column $\left(m^{\prime}, h^{\prime}\right)$ is equal to zero if $m \neq m^{\prime}$ or $h^{\prime}>h$, and is

\footnotetext{
${ }^{1}$ The authors thank the referee for bringing this reference to their attention, and also for several other helpful suggestions and remarks.
} 
otherwise equal to

$$
\lambda_{m, e_{m}-1-\left(h-h^{\prime}\right)}\left(\begin{array}{c}
e_{m}-1-\left(h-h^{\prime}\right) \\
h^{\prime}
\end{array}\right) .
$$

One can also check that

$$
\Phi=\Lambda \mathbf{R} .
$$

We note that $\mathbf{M}_{\mathbf{0}}$ does not depend on the values of $\lambda_{m, h}$. Furthermore, after factoring out various powers of the roots $r_{m}$, it is column-equivalent to the transpose of the matrix whose determinant was computed in Theorem 10. Hence, since we are assuming the $r_{m}$ to be distinct and nonzero, $\mathbf{M}_{\mathbf{0}}$ is nonsingular. Thus we can write

$$
\mathbf{M}_{\mathbf{0}}{ }^{-1} \mathbf{F}=\boldsymbol{\Lambda} \mathbf{R}=\boldsymbol{\Phi},
$$

and the lemma follows.

As an illustration of the previous lemma and its proof, consider the function

$$
F(n)=a r_{1}^{n}+b n r_{1}^{n}+c n^{2} r_{1}^{n}+d r_{2}^{n}+e n r_{2}^{n} .
$$

Then

$$
\begin{aligned}
\Phi_{0}^{1}(n) & =a r_{1}^{n}+b n r_{1}^{n}+c n^{2} r_{1}^{n} \\
\Phi_{1}^{1}(n) & =b r_{1}^{n}+2 c n r_{1}^{n} \\
\Phi_{2}^{1}(n) & =c r_{1}^{n} \\
\Phi_{0}^{2}(n) & =d r_{2}^{n}+e n r_{2}^{n} \\
\Phi_{1}^{2}(n) & =e r_{2}^{n} .
\end{aligned}
$$

We have

$$
\begin{aligned}
\mathbf{M R} & =\left(\begin{array}{ccccc}
a & b & c & d & e \\
(a+b+c) r_{1} & (b+2 c) r_{1} & c r_{1} & (d+e) r_{2} & e r_{2} \\
(a+2 b+4 c) r_{1}^{2} & (b+4 c) r_{1}^{2} & c r_{1}^{2} & (d+2 e) r_{2}^{2} & e r_{2}^{2} \\
(a+3 b+9 c) r_{1}^{3} & (b+6 c) r_{1}^{3} & c r_{1}^{3} & (d+3 e) r_{2}^{3} & e r_{2}^{3} \\
(a+4 b+16 c) r_{1}^{4} & (b+8 c) r_{1}^{4} & c r_{1}^{4} & (d+4 e) r_{2}^{4} & e r_{2}^{4}
\end{array}\right)\left(\begin{array}{c}
r_{1}^{n} \\
n r_{1}^{n} \\
n^{2} r_{1}^{n} \\
r_{2}^{n} \\
n r_{2}^{n}
\end{array}\right) \\
& =\left(\begin{array}{c}
F(n) \\
F(n+1) \\
F(n+2) \\
F(n+3) \\
F(n+4)
\end{array}\right)
\end{aligned}
$$

and

$$
\mathbf{M}=\mathbf{M}_{\mathbf{0}} \boldsymbol{\Lambda}=\left(\begin{array}{ccccc}
0 & 0 & 1 & 0 & 1 \\
r_{1} & r_{1} & r_{1} & r_{2} & r_{2} \\
4 r_{1}^{2} & 2 r_{1}^{2} & r_{1}^{2} & 2 r_{2}^{2} & r_{2}^{2} \\
9 r_{1}^{3} & 3 r_{1}^{3} & r_{1}^{3} & 3 r_{2}^{3} & r_{2}^{3} \\
16 r_{1}^{4} & 4 r_{1}^{4} & r_{1}^{4} & 4 r_{2}^{4} & r_{2}^{4}
\end{array}\right)\left(\begin{array}{ccccc}
c & 0 & 0 & 0 & 0 \\
b & 2 c & 0 & 0 & 0 \\
a & b & c & 0 & 0 \\
0 & 0 & 0 & e & 0 \\
0 & 0 & 0 & d & e
\end{array}\right)
$$

Also

$$
\mathbf{\Phi}=\left(\begin{array}{c}
\Phi_{2}^{1}(n) \\
\Phi_{1}^{1}(n) \\
\Phi_{0}^{1}(n) \\
\Phi_{1}^{2}(n) \\
\Phi_{0}^{1}(n)
\end{array}\right)=\left(\begin{array}{ccccc}
c & 0 & 0 & 0 & 0 \\
b & 2 c & 0 & 0 & 0 \\
a & b & c & 0 & 0 \\
0 & 0 & 0 & e & 0 \\
0 & 0 & 0 & d & e
\end{array}\right)\left(\begin{array}{c}
r_{1}^{n} \\
n r_{1}^{n} \\
n^{2} r_{1}^{n} \\
r_{2}^{n} \\
n r_{2}^{n}
\end{array}\right)=\mathbf{\Lambda} \mathbf{R} .
$$


Thus the relation $\boldsymbol{\Phi}=\mathbf{M}_{\mathbf{0}}{ }^{-1} \mathbf{F}$ expresses each $\Phi_{h}^{m}(n)$ as a linear combination of $F(n), \ldots, F(n+4)$, with coefficients that do not depend on $a, b, c, d$, and $e$.

To prove our main result, we also need the following lemma.

Lemma 22. If $p \geq 0$, define $S_{p}(n, x)=\sum_{j=0}^{n-1} j^{p} x^{j}$. If $x \neq 1$, then

$$
\begin{aligned}
S_{p}(n, x) & =B_{p}(x)-x^{n}\left(\sum_{k=0}^{p}\left(\begin{array}{l}
p \\
k
\end{array}\right) n^{k} B_{p-k}(x)\right) \\
& =B_{p}(x)-x^{n}\left(\left.(n+Q)^{p}\right|_{Q^{k} \rightarrow B_{k}(x)}\right),
\end{aligned}
$$

where $B_{k}(x)=A_{k}(x) /(1-x)^{k+1}$, and $A_{k}(x)$ denotes the Eulerian polynomial of degree $k$. Here, the notation $Q^{k} \rightarrow B_{k}(x)$ means "replace $Q^{k}$ by $B_{k}(x)$ throughout". If $x=1$, then $S_{p}(n, x)$ is a (well-known) polynomial of degree $p+1$.

Proof. The identity

$$
\sum_{j=0}^{\infty} j^{p} x^{j}=\frac{A_{p}(x)}{(1-x)^{p+1}}
$$

is classical (see e.g. [1]), and immediately implies the relation $x B_{p}^{\prime}(x)=B_{p+1}(x)$, as a formal power series identity. The lemma now follows easily by induction.

The next lemma provides an explicit form for our indefinite sums, expressing the result in terms of the functions $\Phi_{h}^{m}(n)$ defined in Lemma 21.

Lemma 23. Suppose that for each $i=1, \ldots, k$,

$$
F_{i}(n)=\sum_{m=1}^{d(i)} \sum_{h=0}^{e_{m}^{(i)}-1} \lambda_{m, h}^{(i)} n^{h}\left(r_{m}^{(i)}\right)^{n}
$$

with $\lambda_{m, e_{m}^{(i)}-1}^{(i)} \neq 0$ for all $i$ and $m$. For $1 \leq i \leq k$, let $\delta_{i, m}=e_{m}^{(i)}-1$. Then

$$
\sum_{j=0}^{n-1} F_{1}(j) F_{2}(j) \cdots F_{k}(j)=\Psi(n)+S(0)-S(n),
$$

where $\Psi(n)$ is a polynomial, and

$$
\begin{aligned}
S(n)= & \sum_{r_{m_{1}}^{(1)} \cdots r_{m_{k}}^{(k)} \neq 1} \sum_{s=0}^{\delta_{1, m_{1}}+\cdots+\delta_{k, m_{k}}} B_{s}\left(r_{m_{1}}^{(1)} \ldots r_{m_{k}}^{(k)}\right) \\
& \times\left\{\sum_{t_{1}+\cdots+t_{k}=s} \Phi_{t_{1}}^{1, m_{1}}(n) \cdots \Phi_{t_{k}}^{k, m_{k}}(n)\right\} .
\end{aligned}
$$

If no product of the form $r_{m_{1}}^{(1)} \cdots r_{m_{k}}^{(k)}$ equals 1 , then $\Psi(n) \equiv 0$; otherwise $\Psi(n)$ has degree at most $1+\sum_{i} \Delta(i)$, where $\Delta(i)=\max _{1 \leq j \leq d(i)}\left(e_{j}^{(i)}-1\right)$, as defined in Theorem 17. In formula (40), $B_{s}(x)=A_{s}(x) /(1-x)^{s+1}$, as defined in Lemma 22 , and for $\ell=1, \ldots, k$,

$$
\Phi_{t}^{\ell, m_{\ell}}(n)=\sum_{i=t}^{\delta_{\ell, m_{\ell}}}\left(\begin{array}{l}
i \\
t
\end{array}\right) \lambda_{m, i}^{(\ell)} n^{i-t}\left(r_{m_{\ell}}^{(\ell)}\right)^{n}
$$

as defined in Lemma 21. 
Note that, in expression (40), the coefficients $B_{s}\left(r_{m_{1}}^{(1)} \ldots r_{m_{k}}^{(k)}\right)$ depend only on the roots $r_{m}^{(i)}$, and hence by Lemma 21, $S(n)$ is independent of the initial conditions of the $F_{i}$.

Proof. To simplify notation, we will first consider the case where the $F_{i}$ each have a single root, i.e.,

$$
F_{i}(n)=\left(\lambda_{0}^{(i)}+\lambda_{1}^{(i)} n+\cdots+\lambda_{\delta_{i}}^{(i)} n^{\delta_{i}}\right)\left(r^{(i)}\right)^{n}
$$

where $r^{(i)}$ is a root of multiplicity $e^{(i)}=\delta_{i}+1$. We have

$$
\begin{aligned}
\sum_{j=0}^{n-1} F_{1}(j) \cdots F_{k}(j) & =\sum_{j=0}^{n-1}\left(\sum_{i=0}^{\delta_{1}} \lambda_{i}^{(1)} j^{i}\left(r^{(1)}\right)^{j}\right) \cdots\left(\sum_{i=0}^{\delta_{k}} \lambda_{i}^{(k)} j^{i}\left(r^{(k)}\right)^{j}\right) \\
& =\sum_{j=0}^{n-1} \sum_{s=0}^{\delta_{1}+\cdots+\delta_{k}} \sum_{i_{1}+\cdots+i_{k}=s} \lambda_{i_{1}}^{(1)} \cdots \lambda_{i_{k}}^{(k)} j^{s}\left(r^{(1)} \cdots r^{(k)}\right)^{j} \\
& =\sum_{s=0}^{\delta_{1}+\cdots+\delta_{k}} \sum_{i_{1}+\cdots+i_{k}=s} \lambda_{i_{1}}^{(1)} \cdots \lambda_{i_{k}}^{(k)} \sum_{j=0}^{n-1} j^{s}\left(r^{(1)} \cdots r^{(k)}\right)^{j}
\end{aligned}
$$

Now for any integer $p \geq 0$, define

$$
H_{p}(n, x)=x^{n} \sum_{k=0}^{p}\left(\begin{array}{l}
p \\
k
\end{array}\right) n^{k} B_{p-k}(x)
$$

where $B_{k}(x)$ is as defined in Lemma 22. Thus, by that lemma, we have

$$
\sum_{j=0}^{n-1} j^{p} x^{j}=H_{p}(0, x)-H_{p}(n, x)=\left.x^{n}(n+Q)^{p}\right|_{Q^{k} \rightarrow B_{k}(x)},
$$

for any $x \neq 1$. Continuing with the above calculation, we obtain

$$
\begin{aligned}
& \sum_{j=0}^{n-1} F_{1}(j) \cdots F_{k}(j) \\
& \quad=\sum_{s=0}^{\delta_{1}+\cdots+\delta_{k}} \sum_{i_{1}+\cdots+i_{k}=s} \lambda_{i_{1}}^{(1)} \cdots \lambda_{i_{k}}^{(k)}\left(H_{s}\left(0, r^{(1)} \cdots r^{(k)}\right)-H_{s}\left(n, r^{(1)} \cdots r^{(k)}\right)\right)
\end{aligned}
$$

provided that $r^{(1)} \cdots r^{(k)} \neq 1$; otherwise the sum is equal to $\Psi(n)$, a polynomial of degree at most equal to $1+\sum_{i} \delta_{i}=1+\sum_{i}\left(e^{i}-1\right)$. In the former case, write

$$
S(n)=\sum_{s=0}^{\delta_{1}+\cdots+\delta_{k}} \sum_{i_{1}+\cdots+i_{k}=s} \lambda_{i_{1}}^{(1)} \cdots \lambda_{i_{k}}^{(k)} H_{s}\left(n, r^{(1)} \cdots r^{(k)}\right)
$$

so that

$$
\sum_{j=0}^{n-1} F_{1}(j) \cdots F_{k}(j)= \begin{cases}S(0)-S(n) & \text { if } r^{(1)} \cdots r^{(k)} \neq 1, \text { and } \\ \Psi(n) & \text { otherwise. }\end{cases}
$$


Using the alternate form in (41), we can write

$$
\begin{aligned}
S(n)= & \left.\sum_{s=0}^{\delta_{1}+\cdots+\delta_{k}} \sum_{i_{1}+\cdots+i_{k}=s} \lambda_{i_{1}}^{(1)} \cdots \lambda_{i_{k}}^{(k)}\left(r^{(1)} \cdots r^{(k)}\right)^{n}(n+Q)^{s}\right|_{Q^{s} \rightarrow B_{s}\left(r^{(1)} \ldots r^{(k)}\right)} \\
= & \left.\left(\sum_{i=0}^{\delta_{1}} \lambda_{i}^{(1)}(n+Q)^{i}\right)\left(r^{(1)}\right)^{n} \cdots\left(\sum_{i=0}^{\delta_{k}} \lambda_{i}^{(k)}(n+Q)^{i}\right)\left(r^{(k)}\right)^{n}\right|_{Q^{s} \rightarrow B_{s}\left(r^{(1)} \ldots r^{(k)}\right)} \\
= & \left(\sum_{i=0}^{\delta_{1}} \lambda_{i}^{(1)} \sum_{u=0}^{i}\left(\begin{array}{c}
i \\
u
\end{array}\right) n^{i-u} Q^{u}\right)\left(r^{(1)}\right)^{n} \\
& \left.\ldots\left(\sum_{i=0}^{\delta_{k}} \lambda_{i}^{(k)} \sum_{u=0}^{i}\left(\begin{array}{c}
i \\
u
\end{array}\right) n^{i-u} Q^{u}\right)\left(r^{(k)}\right)^{n}\right|_{Q^{s} \rightarrow B_{s}\left(r^{(1)} \ldots r^{(k)}\right)} \\
= & \left(\sum_{u=0}^{\delta_{1}} \sum_{i=u}^{\delta_{1}}\left(\begin{array}{c}
i \\
u
\end{array}\right) \lambda_{i}^{(1)} n^{i-u}\left(r^{(1)}\right)^{n} Q^{u}\right) \\
& \left.\cdots\left(\sum_{u=0}^{\delta_{k}} \sum_{i=u}^{\delta_{k}}\left(\begin{array}{c}
i \\
u
\end{array}\right) \lambda_{i}^{(k)} n^{i-u}\left(r^{(k)}\right)^{n} Q^{u}\right)\right|_{Q^{s} \rightarrow B_{s}\left(r^{(1)} \ldots r^{(k)}\right)} \\
= & \left.\left(\sum_{u=0}^{\delta_{1}} \Phi_{u}^{1}(n) Q^{u}\right) \cdots\left(\sum_{v=0}^{\delta_{k}} \Phi_{v}^{k}(n) Q^{v}\right)\right|_{Q^{s} \rightarrow B_{s}\left(r^{(1)} \ldots r^{(k)}\right)}
\end{aligned}
$$

Combining this last expression with (42) yields the statement of Lemma 23 in the case where each recurrence has a single root. For the general case (i.e., when there are multiple roots for each $F_{i}$ ), one can collect terms in the expansion of $F_{1}(j) F_{2}(j) \cdots F_{k}(j)$ corresponding to each choice $r_{m_{1}}, r_{m_{2}}, \ldots, r_{m_{k}}$ of a sequence of roots from each $F_{i}$, and an expression for $K(n)$ of the form (39) results, with $S(n)$ as in (40). This completes the proof of Lemma 23.

Combining Lemmas 21 and 23, we obtain the following theorem, which is the main result of this section.

Theorem 24. If $F_{1}(n), F_{2}(n), \ldots, F_{k}(n)$ satisfy the hypotheses of Lemma 23, then the sum

$$
\sum_{j=0}^{n-1} F_{1}\left(b_{1} j+c_{1}\right) F_{2}\left(b_{2} j+c_{2}\right) \cdots F_{k}\left(b_{k} j+c_{k}\right)
$$

may be expressed as $\Psi(n)-S(n)$, where $\Psi(n)$ is a polynomial and $S(n)$ is a linear combination of monomials of the form

$$
F_{1}\left(b_{1} n+i_{1}\right) F_{2}\left(b_{2} n+i_{2}\right) \cdots F\left(b_{k} n+i_{k}\right) \quad\left(0 \leq i_{\nu} \leq D(\nu)-1 ; 1 \leq \nu \leq k\right)
$$

with $D(\nu)=\sum_{m} e_{m}^{(\nu)}$, such that the coefficients of that linear combination are independent of the initial conditions of the $F_{i}$. If no product $\left(r_{m_{1}}^{(1)}\right)^{b_{1}} \cdots\left(r_{m_{k}}^{(k)}\right)^{b_{k}}$ of the associated roots equals 1 , then $\Psi(n)$ is a constant; otherwise it has degree at most $1+\sum_{i} \Delta(i)$. In general, $\Psi(n)$ will depend on the initial conditions of the $F_{i}$. 


\section{MORE EXAMPLES}

We will give some examples illustrating the results in the previous two sections.

8.1. A mixed convolution. Let $F(n)$ denote the $n$th Fibonacci number, and let $G(n)$ be defined by the subword-avoiding recurrence (13) with $A=3$, in other words $G(0)=1, G(1)=3, G(2)=9$, and $G(n)=3 G(n-1)-G(n-3)$ for $n>2$. Then we have the following identity:

$\sum_{j=0}^{n} j F(j) G(n-j)=18 G(n+1)-(9 G(n)+5 G(n+2)+3 F(n)+n F(n)+n F(n+1))$.

The target monomials in this case are

$$
F(n), n F(n), F(n+1), n F(n+1), G(n), G(n+1), G(n+2),
$$

and the (unique) solution is obtained by solving a system of 7 equations in 7 unknowns. Here we are applying Theorem 17 in the case where no product of the form $\prod r_{m}^{b}$ equals 1 , with $F_{1}(n)=n, F_{2}(n)=F(n)$, and $F_{3}(n)=G(n)$. The sum is $C$-finite of degree 7, and this degree achieves the bounds given in Corollaries 19 and 20 .

8.2. The independence property. We will give two examples of indefinite summations illustrating the phenomena described in Theorems 4 and 24, Consider the sum $\sum_{j=0}^{n-1} F(j)^{3}$, where $F(n)$ satisfies the Fibonacci recurrence with initial conditions $F(0)=p, F(1)=1$. The target monomials are

$$
\text { 1, } F(n)^{3}, F(n)^{2} F(n+1), F(n) F(n+1)^{2}, F(n+1)^{3},
$$

and we obtain the identity

$$
\sum_{j=0}^{n-1} F(j)^{3}=\frac{1}{2}\left(1-3 p+3 p^{3}\right)-\frac{3}{2} F(n)^{3}+\frac{3}{2} F(n) F(n+1)^{2}-\frac{1}{2} F(n+1)^{3},
$$

which is of the form $S(0)-S(n)$ in the notation of Theorem 24 Since the associated roots are distinct and no product equals 1 , this case is covered by Theorem 4 .

Next consider the sum $\sum_{j=0}^{n-1} F(j)^{4}$, for which we now have products of roots equal to 1 . The target monomials are

$$
\text { 1, } n F(n)^{4}, F(n)^{3} F(n+1), F(n)^{2} F(n+1)^{2}, F(n) F(n+1)^{3}, F(n+1)^{4},
$$

and we obtain the general solution

$$
\begin{aligned}
\frac{1}{25}\left(A_{0}+A_{1} n+\right. & 52 F(n)^{3} F(n+1)-22 F(n)^{2} F(n+1)^{2} \\
& \left.-36 F(n) F(n+1)^{3}+19 F(n+1)^{4}\right) \\
+ & K\left(\left(-1+p+p^{2}\right)^{2}-\left(F(n+1)^{2}-F(n)^{2}-F(n) F(n+1)\right)^{2}\right)
\end{aligned}
$$

where

$$
A_{0}=-19+36 p+22 p^{2}-52 p^{3}, \quad A_{1}=6\left(-1+p+p^{2}\right)^{2},
$$

and $K$ is an arbitrary constant. If $p^{2}+p-1 \neq 0$, the polynomial term $\Psi(n)$ in Theorem 24 will always have degree 1. 
8.3. Partial summation of series. Consider the sum

$$
\sum_{j=0}^{n-1} F(j) x^{j}
$$

where $F(n)$ is the $n$th Fibonacci number and $x$ is an indeterminate. The summand is a product of two $C$-finite sequences, one of degree two and the other of degree one. Following Theorem 17] we construct a list of target monomials $1, F(n) x^{n}$, and $F(n+1) x^{n}$, and from these we obtain the identity

$$
\sum_{j=0}^{n-1} F(j) x^{j}=\frac{x}{1-x-x^{2}}-x^{n}\left(\frac{1-x}{1-x-x^{2}} F(n)+\frac{x}{1-x-x^{2}} F(n+1)\right) .
$$

This identity quantifies the remainder term in the Fibonacci generating function (an equivalent result appears as problem 1.2.8.21 in [5]). Our approach can be easily extended; for example, using $1, F(n)^{2} x^{n}, F(n) F(n+1) x^{n}$, and $F(n+1)^{2} x^{n}$ as target monomials and solving four equations in four unknowns, we obtain the partial summation formula

$$
\sum_{j=0}^{n-1} F(j)^{2} x^{j}=\frac{x(1-x)}{1-2 x-2 x^{2}+x^{3}}-x^{n} R_{n}(x),
$$

where

$$
R_{n}(x)=\frac{\left(1-2 x-x^{2}\right) F(n)^{2}+2 x^{2} F(n) F(n+1)+x(1-x) F(n+1)^{2}}{1-2 x-2 x^{2}+x^{3}} .
$$

The first term in (43) is the full generating function for squares of Fibonacci numbers. A formula for the full generating function for all powers $p$ appears in [8] (see also [5], problem 1.2.8.30).

We note that, to obtain (43) and (44) by this method, it was only necessary to know the first four values of the sum, and also that $F$ satisfies some 2-term recurrence with constant coefficients.

8.4. The degree of the polynomial multiplier. In Theorem 17 we gave a set of monomials in terms of which the sum can be expressed, in the general case of repeated roots. In those monomials a polynomial factor $\psi_{i_{1} \ldots i_{k}}$ appears, and the degree of that polynomial was found to be at most $1+\sum\left\{\Delta(i): a_{i}=0\right\}$. We remark here that the $C$-finite function $F(n)=n^{p}$, for positive integer $p$, shows that this upper bound can be achieved. For here we have

$k=1, d(1)=1, r_{1}^{(1)}=1, e_{1}^{(1)}=p+1, \Delta(1)=p, D(1)=p+1,\left(a_{1}, b_{1}, c_{1}\right)=(0,1,0)$.

The monomials in the list (24) are all of degree $p$, and those in the list (25) are of degree equal to the degree of $\psi_{1}(n)$. The maximum allowable degree of the latter is $1+\Delta(1)=p+1$, and in this case $\psi$ is of degree $p+1$ since the sum obviously is also.

8.5. An example from the theory of partitions. Our algorithm for summation of $C$-finite sequences can sometimes have by-products that are more interesting than the particular problem being solved. A small example of this is given here. Suppose 
$p_{5}(n)$ is the number of partitions of $n$ into $\leq 5$ parts. Then $\sum_{n \geq 0} p_{5}(n) z^{n}=$ $\left((1-z)\left(1-z^{2}\right)\left(1-z^{3}\right)\left(1-z^{4}\right)\left(1-z^{5}\right)\right)^{-1}$, so $p_{5}$ is $C$-finite of degree $\leq 15$. We asked our Mathematica program [4] to find $f(n)=\sum_{0 \leq j \leq n-1} p_{5}(j)$. In addition to giving the answer, a number of the arbitrary constants that are used to form linear combinations with target monomials were left unassigned, and since the coefficient of every such unassigned constant must of course vanish, one has found an identity. On this occasion we chose one symmetrical looking such identity from the output, namely

$$
\begin{gathered}
p_{5}(n-10)+4 p_{5}(n-9)+9 p_{5}(n-8)+15 p_{5}(n-7) \\
+20 p_{5}(n-6)+22 p_{5}(n-5)+20 p_{5}(n-4) \\
+15 p_{5}(n-3)+9 p_{5}(n-2)+4 p_{5}(n-1)+p_{5}(n)=\left(\begin{array}{c}
n+4 \\
4
\end{array}\right) .
\end{gathered}
$$

The coefficients on the left side are recognized as the numbers of permutations of 5 letters that have $k$ inversions. From this one might suspect that we have generally,

$$
\sum_{j} b(k, j) p_{k}(n-j)=\left(\begin{array}{c}
n+k-1 \\
n
\end{array}\right),
$$

where $b(k, j)$ is the number of $k$-permutations that have exactly $j$ inversions and $p_{k}(m)$ is the number of partitions of $m$ into parts $\leq k$. A proof of this identity by generating functions is quite trivial. Here is a bijective proof, that is, a bijection between pairs consisting of a permutation of $k$ letters with $j$ inversions and a partition of $n-j$ into $\leq k$ parts, on the one hand, and one of the $\left(\begin{array}{c}n+k-1 \\ n\end{array}\right)$ compositions of $n$ into $k$ nonnegative parts, on the other. Take such a composition $X$ of $n$ into $k$ parts. Perform a "modified bubble sort," whereby whenever one sees an adjacent pair $x y$ with $x<y$, it is replaced by $(y-1) x$. Keep doing this until there are no adjacent pairs $x<y$, i.e., until a partition $\lambda$ (perhaps followed by 0 's) is obtained. Call the resulting permutation of positions $\sigma$. Then $\lambda$ and $\sigma$ are uniquely determined by $X$, and the correspondence is bijective.

We do not claim novelty for this result or its proof, but offer it only as an example of the usefulness that our algorithms can have in the discovery process.

\section{REFERENCES}

[1] L. Comtet, Advanced Combinatorics, D. Reidel, Dordrecht, 1974. MR0460128 (57:124)

[2] R. P. Flowe, G. A. Harris, A note on generalized Vandermonde determinants, SIAM J. Matrix Anal. Appl. 14 (1993), no. 4, 1146-1151. MR.1238929 (94j:15005)

[3] Charles Jordan, Calculus of Finite Differences, Chelsea, New York, 1950.

[4] Curtis Greene, Herbert S. Wilf, CFSum.nb, (Mathematica notebook), <http://www.haverford.edu/math/cgreene/cfsum.nb>, <http://www.math.upenn.edu/ $\sim$ wilf/website/cfsum.nb>.

[5] Donald E. Knuth, The Art of Computer Programming, Addison-Wesley, Reading, MA, 1969, Vol. 1, p. 84 (exercises 1.2.8.21 and 1.2.8.30), and p. 491, p. 492 (solutions). MR0378456 (51:14624)

[6] Christian Krattenthaler, Advanced Determinant Calculus, Séminaire Lotharingien Combin. 42 ("The Andrews Festschrift") (1999), Article B42q, 67 pp. MR 1701596 (2002i:05013)

[7] Marko Petkovšek, Herbert S. Wilf, and Doron Zeilberger, $A=B$, A K Peters Ltd., Wellesley, MA, 1996. MR 1379802 (97j:05001)

[8] J. Riordan, Generating functions for powers of Fibonacci numbers, Duke. Math. J. 29 (1962), 5-12. MR0132023 (24:A1870) 
[9] David L. Russell, Sums of products of terms from linear recurrence sequences, Discrete Math 28 (1979), 65-79. MR 0542937 (82j:10021)

[10] Doron Zeilberger, A holonomic systems approach to special functions identities, J. Comput. Appl. Math. 32 (1990), no. 3, 321-368. MR1090884 (92b:33014)

Department of Mathematics, Haverford College, Haverford, Pennsylvania 190411392

E-mail address: cgreene@haverford.edu

Department of Mathematics, University of Pennsylvania, Philadelphia, Pennsylvania 19104-6395

E-mail address: wilf@math.upenn.edu 